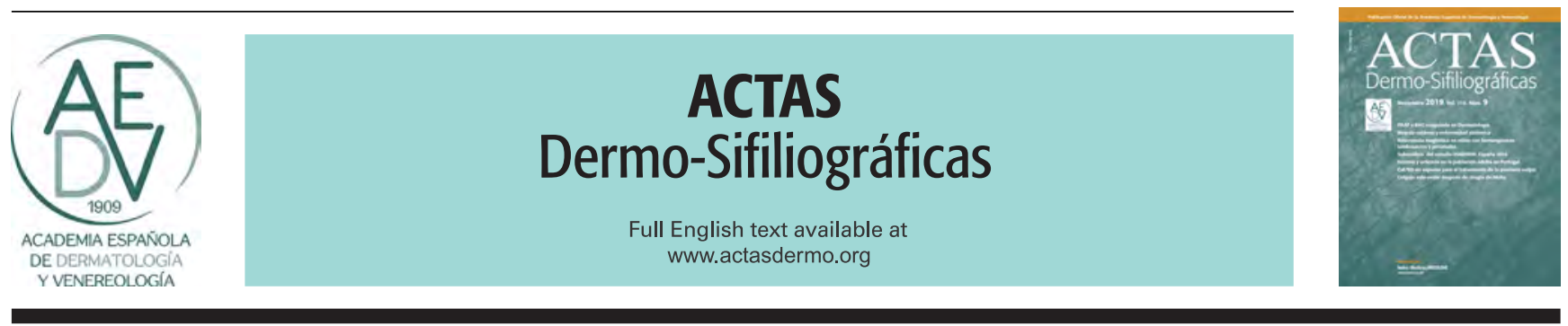

\title{
Revisión sistemática de las terapias con luz en el tratamiento de la hidradenitis supurativa
}

\author{
T. Gracia Cazaña ${ }^{a, *}$, L.V. Berdel Díaz ${ }^{\mathrm{b}}$, J.I. Martín Sánchez ${ }^{\mathrm{c}}$, I. Querol Nasarre $^{\mathrm{b}}$ \\ e Y. Gilaberte ${ }^{d}$
}

\author{
a Unidad de Dermatología, Hospital de Barbastro, Barbastro, Huesca, España \\ b Departamento de Medicina, Psiquiatría y Dermatología, Facultad de Medicina, Universidad de Zaragoza, Zaragoza, España \\ c Decisiones Basadas en la Evidencia, Instituto Aragonés de Ciencias de la Salud, Zaragoza, España \\ ' Servicio de Dermatología, Hospital Universitario Miguel Servet, IIS Aragón, Zaragoza, España
}

Recibido el 17 de marzo de 2019; aceptado el 22 de abril de 2019

Disponible en Internet el 20 de diciembre de 2019

\section{PALABRAS CLAVE \\ Hidradenitis \\ supurativa; \\ Terapia fotodinámica; \\ Láser; \\ Luz intensa pulsada}

\section{KEYWORDS}

Hidradenitis

suppurativa;

Photodynamic

therapy;

Laser;

Intense pulsed light
Resumen La terapia con luz es una alternativa en el tratamiento de la hidradenitis supurativa. El objetivo de este estudio fue evaluar la eficacia, la efectividad y la seguridad de las distintas modalidades de terapia con luz en la hidradenitis supurativa mediante una revisión sistemática que actualiza la realizada en 2015 procedente de la Cochrane Library. Se identificaron los estudios mediante una búsqueda electrónica en las bases de datos: MEDLINE, EMBASE, Centre for Reviews and Dissemination database de la Universidad de York, Cochrane Database of Systematic Reviews, Cochrane Skin Group Specialised Skin Register, Centre of Evidence Based Dermatology de la Universidad de Nottingham y TESEO; finalmente se incluyeron 6 series de casos, 3 revisiones sistemáticas y 2 ensayos clínicos de pacientes tratados con terapia fotodinámica convencional o intralesional, láser $\mathrm{Nd}$ :YAG, $\mathrm{CO}_{2}$ o diodo y luz intensa pulsada, con un total de 248 pacientes tratados.

(c) 2019 AEDV. Publicado por Elsevier España, S.L.U. Este es un artículo Open Access bajo la licencia CC BY-NC-ND (http://creativecommons.org/licenses/by-nc-nd/4.0/).

\section{Systematic Review of Light-Based Treatments for Hidradenitis Suppurativa}

Abstract Treatment options for hidradenitis suppurativa include light-based therapy. This systematic review aimed to update our understanding of the efficacy, effectiveness and safety of these treatments for hidradenitis suppurativa by evaluating the literature published since the 2015 Cochrane review on this topic. We conducted an electronic search of the following databases: MEDLINE, EMBASE, University of York Centre for Reviews and Dissemination

\footnotetext{
* Autor para correspondencia.

Correo electrónico: tamgracaz@gmail.com (T. Gracia Cazaña).
} 
database, Cochrane Database of Systematic Reviews, Cochrane Skin Group Specialised Skin Register, University of Nottingham's Centre of Evidence Based Dermatology database, and TESEO. The systematic review included 6 case series, 3 systematic reviews, and 2 clinical trials on the use of conventional and intralesional photodynamic therapy, intense pulsed light therapy, and Nd:YAG, carbon dioxide, and diode laser therapy for hidradenitis suppurative in 248 patients.

(C) 2019 AEDV. Published by Elsevier España, S.L.U. This is an open access article under the CC BY-NC-ND license (http://creativecommons.org/licenses/by-nc-nd/4.0/).

\section{Introducción}

La hidradenitis supurativa (HS) es una enfermedad inflamatoria, crónica y debilitante del folículo pilosebáceo que habitualmente aparece tras la pubertad, en la que subyace un desequilibrio del sistema inmunológico, en una persona predispuesta genéticamente ${ }^{1}$. Su prevalencia oscila entre el 0,1 y el $1 \%$ de la población, siendo más frecuente entre la segunda y la tercera décadas de la vida, y en las mujeres ${ }^{2}$. El tabaquismo, la obesidad, alteraciones endocrinológicas y las fuerzas de fricción o cizallamiento pueden actuar como desencadenantes o agravantes ${ }^{3}$.

La forma más frecuente de presentación clínica son nódulos, abscesos, fístulas supurativas o cicatrices localizados en pliegues, principalmente en las axilas, las ingles y regiones anogenitales ${ }^{4}$. Los síntomas predominantes son dolor, sensación de quemazón y calor local, prurito e hiperhidrosis, que tienen una repercusión negativa en la calidad de vida de los pacientes $^{5}$.

El manejo de la HS es complejo y requiere un abordaje multidisciplinar $^{6}$. Los tratamientos con luz han sido utilizados en los últimos años, de una forma experimental. La terapia fotodinámica (TFD) utiliza un fotosensibilizante que, activado con luz y en presencia de oxígeno, genera especies reactivas del oxígeno que causan destrucción celular. En el caso de la HS, se ha propuesto que el efecto beneficioso de la TFD se basaría en un efecto citotóxico directo sobre las bacterias en biofilm y las glándulas sebáceas, así como por la inducción de una respuesta inmunomoduladora ${ }^{1}$. Cuando los agentes fotosensibilizantes se aplican tópicamente en las lesiones de HS, la inflamación y la presencia de orificios fistulosos y úlceras facilitan su penetración y acúmulo en comparación con la piel normal ${ }^{7}$. La posterior fotoactivación con luz de $630 \mathrm{~nm}$ de longitud de onda, adecuada para excitar tanto al ácido 5-aminolevulínico (ALA) como al azul de metileno (AM), los 2 fotosensibilizantes hasta ahora utilizados, induce una mejoría de las lesiones ${ }^{8}$. La TFD tópica presenta unas ventajas que la convierten en una atractiva alternativa terapéutica por su capacidad de tratar múltiples lesiones simultáneamente, ser poco invasiva, tener pocos efectos adversos y una buena tolerancia?.

La terapia con luz pulsada intensa (IPL) utiliza una luz no coherente de alto espectro y alta energía. Su principio básico consiste en la absorción de fotones por cromóforos exógenos o endógenos cutáneos. Esta transferencia de energía a las estructuras diana (en la HS, el folículo piloso ocluido) genera calor y la subsiguiente destrucción de la lesión a través de un proceso denominado fototermólisis selectiva ${ }^{10}$. Además, la IPL tiene un efecto antimicrobiano a través de la excitación de las propias porfirinas bacterianas que genera especies reactivas del oxígeno y muerte celular ${ }^{11}$

Los láseres de $\mathrm{Nd}$ :YAG, $\mathrm{CO}_{2}$, alejandrita o diodo también se han utilizado en el tratamiento de la HS con el objetivo de reducir el número de folículos pilosos en áreas proclives al desarrollo de lesiones de la $\mathrm{HS}^{12}$.

Considerando lo expuesto, el objetivo de este estudio es evaluar la eficacia, la efectividad y la seguridad de las distintas modalidades de terapia con luz en el tratamiento de la HS mediante una revisión sistemática. Se dividirá en TFD convencional, TFD intralesional y láseres, sin compararlos entre sí, ya que cada uno presenta un mecanismo de acción diferente, pero dado el escaso número de publicaciones al respecto, se han agrupado en esta revisión de terapias con luz.

\section{Material y método}

\section{Métodos de búsqueda para la localización de los estudios}

Se realizó una búsqueda bibliográfica para identificar todos los trabajos publicados desde agosto de 2015 hasta el 1 de marzo del 2019, sobre el tratamiento de la HS con luz como monoterapia o con luz más fotosensibilizante (TFD). Los resultados se han limitado a estudios realizados en humanos y publicados en español o inglés. Debido a que se ha identificado una revisión sistemática, con la última actualización en agosto de 2015, procedente de la Cochrane Library vía Cochrane Database of Systematic Reviews, se justifica el acotamiento de la fecha de búsqueda para el presente trabajo, ya que el objetivo principal es actualizar la información ya recogida sobre el tema en esta revisión sistemática previa.

Además, se ha llevado a cabo una búsqueda inversa, desde trabajos identificados con fecha anterior a la acotada, realizando una revisión bibliográfica de los mismos.

La identificación de los estudios se ha llevado a cabo mediante la búsqueda en las siguiente bases de datos: MEDLINE vía PubMed, EMBASE, Centre of Review and Dissemination vía Universidad de York, Cochrane Library vía Cochrane Database of Systematic Reviews, Cochrane Skin Group, Centre of Evidence Based Dermatology de la Universidad de Nottingham y TESEO, base de datos de tesis doctorales. Adicionalmente, se ha ampliado la búsqueda 


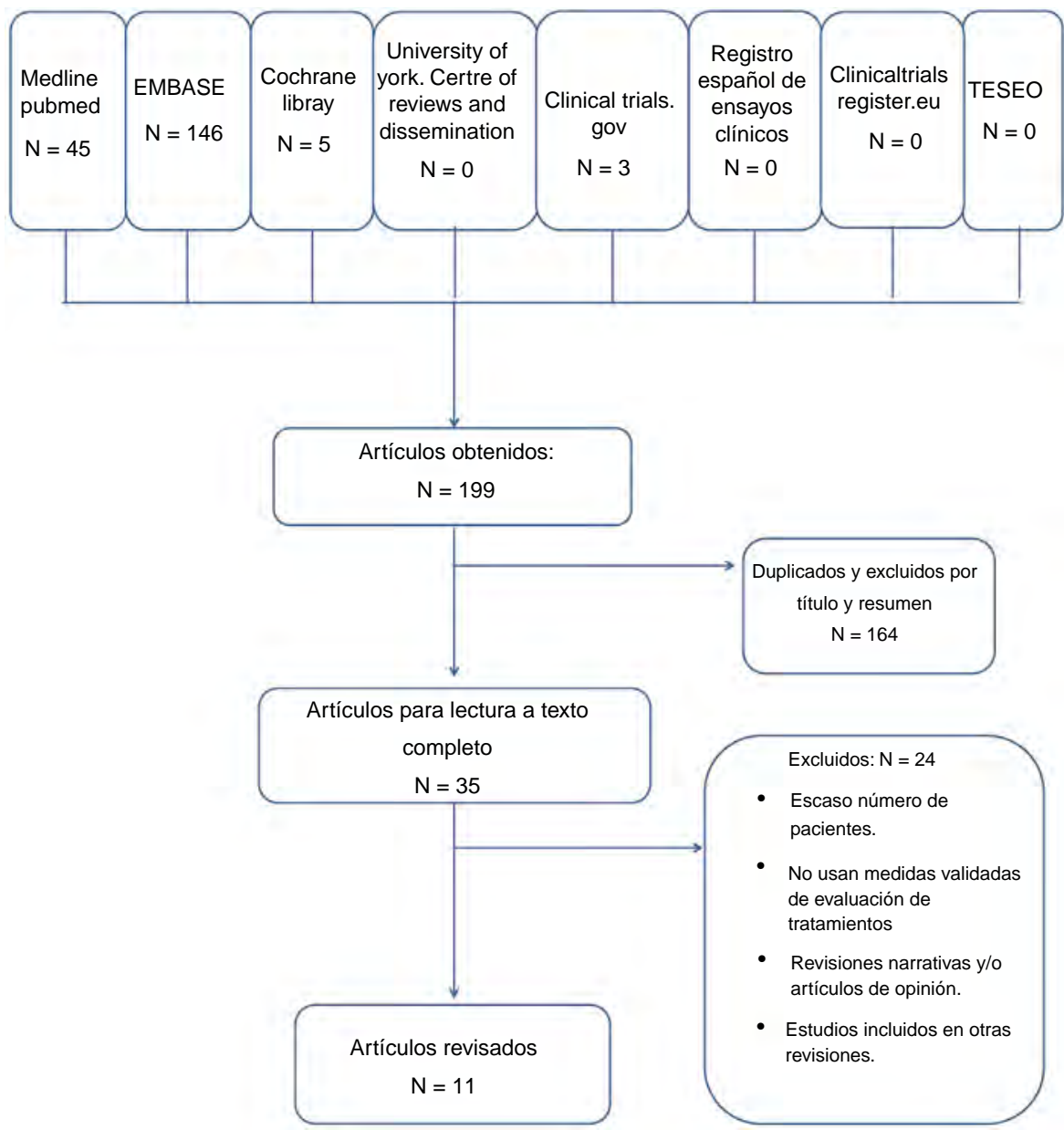

Figura 1 Diagrama de flujo de la búsqueda bibliográfica realizada en la revisión sistemática.

con las siguientes webs electrónicas: ClinicalTrials.gov, reec.aemps.es, Clinicaltrialsregister.eu.

Finalmente, con el objetivo de identificar las revisiones sistemáticas sobre HS, y más concretamente sobre el tratamiento con luz, que se están llevando a cabo en este momento, se ha realizado una búsqueda electrónica en PROSPERO, registro internacional de revisiones sistemáticas.

Los términos de búsqueda que se han introducido para llevar a cabo la búsqueda definitiva de artículos, de acuerdo con el diccionario MeSH y DeSC, han sido: photochemotherapy, photodynamic therapy, hidradenitis suppurativa, suppurative hidradenitis, light, lasers, laser therapy, intense pulsed light therapy.

Criterios de inclusión de los estudios:

- Tipos de estudios: ensayos clínicos controlados y aleatorizados; revisiones sistemáticas; estudios observacionales con grupo control; y estudios observacionales sin grupo control (series de casos).

- Población: pacientes con HS mayores de edad, independientemente del sexo, la etnia, la comorbilidad y el tratamiento previo.

- Para que lo estudios sean considerados, deben incluir pacientes con HS tratados con TFD, cualquier tipo de láser o luz.
- Se aplicará restricción a los siguientes idiomas: español e inglés.

Criterios de exclusión de los estudios:

- Estudios publicados fuera del rango de tiempo establecido.

- Estudios que no utilicen las medidas de evaluación aceptadas y validadas para HS (HiSCR y/o escala Sartorius modificada y/o HsPGA).

- Series de casos con un número de pacientes inferior a 5.

- Editoriales y revisiones narrativas.

\section{Manejo, extracción de datos, síntesis de datos. Análisis de la calidad metodológica}

Los resultados obtenidos por las búsquedas fueron descargados a un procesador de referencias bibliográficas (Mendeley ${ }^{\circledR}$ ), donde se han filtrado los trabajos en función del título y el resumen. Durante este proceso, todas las referencias duplicadas, descartadas y las seleccionadas han sido registradas a través de la realización de un diagrama de flujo (fig. 1). La búsqueda no ha sido ciega en cuanto a autores, revista o institución.

Se ha consultado la escala de Jadad $^{13}$ para los ensayos aleatorizados, y el análisis crítico se ha basado en la guía CONSORT ${ }^{14}$. Para sintetizar los datos se ha utilizado la 
herramienta OSTEBA ${ }^{15}$, ya que permite valorar la calidad de la evidencia de los diversos tipos de estudios, así como elaborar tablas de síntesis de resultados.

\section{Resultados}

\section{Estudios incluidos y excluidos}

De los 199 registros obtenidos, se han excluido 164 tras la evaluación del título y el resumen, y por duplicidad en las bases de datos de la búsqueda. Se han evaluado 35 artículos, en texto completo, de los que se excluyeron 24 por no cumplir los criterios de inclusión o tener un número escaso de pacientes (Anexo incluido en material suplementario) $)^{9,12,16-37}$. Dado el escaso número de ensayos clínicos y revisiones sistemáticas, se han incluido series de casos, pese a que la evaluación de la eficacia y la efectividad en ellos es muy limitada.

Los estudios que se han evaluado son: 6 series de casos, 5 prospectivos y uno retrospectivo, de los que 4 tienen una baja calidad de la evidencia y 2 , una calidad media.

La mayoría de los estudios se realizaron en Europa (España, Reino Unido, Francia e Italia),Estados Unidos y Egipto. Los trabajos restantes son 2 ensayos clínicos de calidad media y 3 revisiones sistemáticas con una alta calidad de la evidencia, que incluyen 4 ensayos clínicos y 5 series de casos sobre el tratamiento con luz, que se analizan en profundidad en este trabajo: Fadel y Tawfik ${ }^{38}$, Highton et al. ${ }^{11}$, Tierney et al. ${ }^{39}$, Calzavara-Pinton et al. ${ }^{40}$, Schweiger et al. ${ }^{41}$, Sotiriou et al. ${ }^{42}$ Mahmoud et al. ${ }^{43}$, Madan et al. ${ }^{44}$ y Xu et al. ${ }^{45}$.

El periodo de seguimiento, el número de pacientes, el número de pérdidas, las características de la población a estudio y la intervención aplicada de los estudios incluidos están resumidos en las tablas 1-3. Uno de los trabajos incluidos ${ }^{2}$ ha sido extraído a través de la búsqueda inversa de artículos por considerarlo relevante para esta revisión.

\section{Tamaño de la muestra}

El número de participantes en los estudios incluidos varió considerablemente, siendo el máximo de $38^{46}$ y el mínimo de $5^{2,42}$, con un total de 248 pacientes tratados. La media del número de participantes fue de 15,5.

\section{Participantes}

Todos los estudios incluyeron adultos mayores de 18 años con diagnóstico clínico de HS, tanto hombres como mujeres.

Un estudio solo incluyó pacientes con HS grado I de Hurley (Zerbinati et al. ${ }^{47}$ ) y otro con Hurley tipo "I (Mahmoud et al. ${ }^{43}$ ). Dos estudios incluyeron HS Hurley tipo I 0 ॥ (Abdel Azim et al. ${ }^{48}$, Fabbrocini et al. ${ }^{49}$ ). Seis estudios exigieron que la gravedad basal de la HS fuera moderada a grave según la clasificación de Hurley (grados II o III) (Andino Navarrete et al. ${ }^{2}$, Agut-Busquet et al. ${ }^{1}$, Highton et al. ${ }^{11}$, Tierney et al. ${ }^{39}$, Xu et al. ${ }^{45}$, Sotiriou et al. ${ }^{42}$ ). Un estudio admitió cualquier nivel de gravedad (grados I, II O III) (Suárez Valladares et al..$^{50}$ ). Finalmente, 5 de ellos no especifican la graduación (Valladares-Narganes et al. ${ }^{51}$, Fadel y Tawfik ${ }^{38}$, Calzavara-Pinton et al. ${ }^{40}$, Madan et al. ${ }^{44}$, Schweiger et al. ${ }^{41}$ ).

\section{Diseño}

Tres de los estudios incluidos en el trabajo son revisiones sistemáticas (Ingram et al. ${ }^{3}$, John et al. ${ }^{52}$ y Robert et al. ${ }^{53}$ ), 2 son ensayos clínicos (Fadel y Tawfik ${ }^{38}$ y Abdel Azim et al. ${ }^{48}$ ) y el resto son series de casos.

En la revisión sistemática de Ingram et al. ${ }^{3}$ se incluyen 3 ensayos clínicos controlados, que investigan la TFD tópica, la IPL y el láser Nd:YAG ${ }^{11,38,39}$. En cuanto al grupo control, Fadel y Tawfik $^{38}$ incluyen un comparador activo, Highton et al. ${ }^{11}$ un grupo sin tratamiento y Tierney et al..$^{39}$ aplican clindamicina al $1 \%$ tópica. En la revisión que llevaron a cabo John et al. ${ }^{52}$ en la que incluyeron 22 estudios, solo 3 tenían una calidad de la evidencia alta, ya incluidos en la revisión de Ingram et al. ${ }^{3}$; el único que no está duplicado, con una calidad de la evidencia moderada y que cumple nuestros criterios de inclusión, es el de Xu et al. ${ }^{45}$, que evalúa el tratamiento con láser Nd:YAG. La revisión sistemática más actualizada es la de Robert et al. ${ }^{53}$, en la que se incluyeron 64 artículos sobre tratamientos no quirúrgicos en $\mathrm{HS}$, tanto tratamiento médico como con luz; de estos últimos, entre los no duplicados tratados con TFD con metilaminolevulinato (MAL) se incluyó el de Calzavara-Pinton et al. ${ }^{40}$, y tratados con ALA al $20 \%$ los de Schweiger et al. ${ }^{41}$ y Sotiriou et al. ${ }^{42}$. Además, en esta revisión se incluyó la serie de casos de Mahmoud et al. ${ }^{43}$ tratados con láser Nd:YAG y los de Madan et al. ${ }^{44}$ tratados con láser de $\mathrm{CO}_{2}$.

Tan solo 2 estudios fueron ensayos clínicos, el de Fadel y Tawfik $^{38}$, que se realizó a través de una búsqueda retrospectiva ya comentada previamente, y el de Abdel Azim et al. ${ }^{48}$, que evaluó el tratamiento combinado con láser de $\mathrm{CO}_{2}$ seguido de $\mathrm{Nd}$ YYAG.

De los trabajos con diseño de serie de casos, uno de ellos estudió la aplicación de TFD tópica con fotosensibilizante ALA al 20\% y láser $635 \mathrm{~nm}$ (Andino Navarrete et al. ${ }^{2}$ ). Tres estudios analizaron la aplicación de TFD intralesional: 2 de ellos con ALA al 5 y al 1\% e iluminación con láser de $630 \mathrm{~nm}^{50,51}$ y otro con $\mathrm{AM}$ al $1 \%$ e iluminación con lámpara de diodo de $630 \mathrm{~nm}$ (Aktilite $\left.{ }^{\circledR}\right)^{1}$.

Los que evaluaron el tratamiento con láser fueron las series de casos de Zerbinati et al. ${ }^{47}$, que estudiaron el tratamiento con láser Nd:YAG, y por último, Fabbrocini et al. ${ }^{49}$, que evaluaron la eficacia del láser diodo en la HS levemoderada.

\section{Medidas de resultados}

En los trabajos incluidos hubo cierta variación en el método de evaluación de los resultados. La mayoría de ellos utilizaron la escala de Sartorius $2003^{2,11,38,39,41-43,45,47,49}$, y 2, la escala de Sartorius modificada ${ }^{1,50,51}$. Agut-Busquet et al. ${ }^{1}$, Abdel Azim et al. ${ }^{48}$, Calzavara-Pinton et al. ${ }^{40}$, Fabbrocini et al. ${ }^{49}$ y Madan et al. ${ }^{44}$ utilizan la escala de evaluación global del médico (PGA).

Seis trabajos evaluaron los cambios en la calidad de vida mediante el índice de calidad de vida dermatológica (DLQI, Finlay 1994$)^{1,2,41,42,49,50}$. Highton et al. ${ }^{11}$ valoran la satisfacción general de los participantes usando una escala de Likert 
Tabla 1 Resumen de los artículos de terapia fotodinámica tópica sola o en combinación con cirugía en hidrosadenitis supurativa incluidos en la revisión sistemática

\begin{tabular}{|c|c|c|c|c|c|c|c|}
\hline Referencia & Estudio & Población & Intervención y seguimiento & Resultados & Conclusiones & Comentarios & $\begin{array}{l}\text { Calidad de la } \\
\text { evidencia }\end{array}$ \\
\hline $\begin{array}{l}\text { Andino Navarrete } \\
\text { et al. } .^{2}, 2014\end{array}$ & $\begin{array}{l}\text { Diseño: serie de } \\
\text { casos, prospectivo } \\
\text { Objetivos: evaluar } \\
\text { la eficacia del } \\
\text { tratamiento, en } \\
\text { pacientes con HS } \\
\text { moderada a grave, } \\
\text { con TFD con ALA y } \\
\text { luz } 635 \mathrm{~nm}, \\
\text { mediante la } \\
\text { puntuación de } \\
\text { Sartorius, calidad } \\
\text { de vida y seguridad }\end{array}$ & $\begin{array}{l}\text { Número de } \\
\text { participantes/grupo: } 5 \\
\text { pacientes } \\
\text { Características } \\
\text { participantes: } \\
\text { pacientes con HS clase } \\
\text { II o III de Hurley. Cuatro } \\
\text { bilateral axilar y una } \\
\text { púbica } \\
\text { Un hombre, } 4 \text { mujeres } \\
\text { Edad media } 26 \pm 5 \\
\text { años. Duración } \\
\text { promedio de la } \\
\text { enfermedad } 4 \pm 1 \text { años }\end{array}$ & $\begin{array}{l}\text { Solución de ALA } 20 \%, 1,5 \mathrm{~h} \text {, } \\
\text { luz de } 635 \mathrm{~nm}, 37 \mathrm{~J} / \mathrm{cm}^{2} \text { e } \\
\text { intensidad } 70 \mathrm{~mW} / \mathrm{cm}^{2} \text { por } \\
\text { sesión } \\
\text { Mínimo } 4 \text { sesiones, cada } 1-2 \\
\text { semanas } \\
\text { Periodo de seguimiento: } \\
\text { control a las } 4 \text { y } 8 \text { semanas } \\
\text { Número de pérdidas: cero }\end{array}$ & $\begin{array}{l}\text { Mejoría significativa del score } \\
\text { de gravedad, DLQI y EVA, } \\
\text { mantenida a las } 8 \text { semanas de } \\
\text { seguimiento } \\
\text { La puntuación de Sartorius } \\
\text { disminuyó de } 35 \pm 5 \text { a } 18 \pm 8 \\
\text { puntos de media } \\
\text { El DLQI disminuyó } 21 \text { puntos } \\
\text { tras } 4 \text { sesiones, mantenido a } \\
\text { las } 8 \text { semanas } \\
\text { La EVA pretratamiento fue } 3 \\
\text { en todos los casos, } \\
\text { disminuyendo a las } 4 \text { y } 8 \\
\text { semanas }(0-1) \text { en todos los } \\
\text { pacientes } \\
\text { El único efecto adverso leve } \\
\text { fue ardor durante la } \\
\text { irradiación en } 2 \text { pacientes }\end{array}$ & $\begin{array}{l}\text { El tratamiento con ALA } 20 \% \\
\text { tópico y luz } 635 \mathrm{~nm} \text { podría ser } \\
\text { efectivo en la HS refractaria, } \\
\text { disminuyendo la gravedad y } \\
\text { mejorando la calidad de vida } \\
\text { de los pacientes }\end{array}$ & $\begin{array}{l}\text { La evidencia es } \\
\text { baja porque la } \\
\text { serie es corta, lo } \\
\text { que condiciona la } \\
\text { validez externa y } \\
\text { la extrapolación a } \\
\text { la población }\end{array}$ & Baja \\
\hline $\begin{array}{l}\text { Fadel y Tawfik }{ }^{38} \\
2015\end{array}$ & $\begin{array}{l}\text { Diseño: ensayo } \\
\text { clínico } \\
\text { aleatorizado, } \\
\text { simple ciego } \\
\text { Objetivos: evaluar } \\
\text { la eficacia y la } \\
\text { seguridad del AM } \\
\text { como } \\
\text { fotosensibilizante } \\
\text { administrado en } \\
\text { gel niosomal para } \\
\text { el tratamiento de } \\
\text { la HS con IPL } \\
\text { Periodo de } \\
\text { realización: } \\
\text { Seis meses (no } \\
\text { especifica mes ni } \\
\text { año) }\end{array}$ & $\begin{array}{l}\text { Número de } \\
\text { participantes/grupo: } \\
\text { se inscribieron } 17 \text { y se } \\
\text { excluyeron } 6 \\
\text { (finalmente hubo } 11 \\
\text { pacientes) } \\
\text { Caracteristicas de los } \\
\text { participantes: } 7 \\
\text { mujeres y } 4 \text { hombres } \\
\text { con una media de } 27,1 \\
\text { años de edad (rango } \\
\text { 17-35) } \\
\text { Los pacientes tenían } \\
\text { lesiones en ambas } \\
\text { axilas (4), lesiones } \\
\text { múltiples en las nalgas } \\
\text { (3), ingle (2) y región } \\
\text { inframamaria (1) } \\
\text { Sin tratamiento } \\
\text { sistémico ni tópico } 2 \\
\text { semanas antes de la } \\
\text { inscripción en el } \\
\text { estudio }\end{array}$ & $\begin{array}{l}\text { Intervención grupo } \\
\text { experimental: TFD tópica con } \\
\text { gel de AM 0,01\% e IPL } 630 \mathrm{~nm} \text {, } \\
20 \mathrm{~ms}, 25 \mathrm{~J} \text {, una cada } 2 \\
\text { semanas } \\
\text { Dos sesiones al mes, hasta } 6 \\
\text { meses } \\
\text { Intervención grupo control: } \\
\text { control intrapaciente un lado } \\
\text { (lado de control). TFD tópica } \\
\text { con gel libre de AM, seguido } \\
\text { de IPL } 630 \mathrm{~nm}, 20 \mathrm{~ms} \text {, } \\
25 \mathrm{~J} / \mathrm{cm}^{2} \text {, una vez cada } 2 \\
\text { semanas } \\
\text { Periodo de seguimiento: los } \\
\text { pacientes fueron seguidos } \\
\text { uno, } 3 \text { y } 6 \text { meses después del } \\
\text { tratamiento } \\
\text { Pérdidas postaleatorización: } \\
\text { ninguna }\end{array}$ & $\begin{array}{l}\text { TFD tópica con gel de AM } \\
0,01 \% \text { e IPL fue superior a la } \\
\text { TFD con gel libre de AM con } \\
\text { una reducción en la } \\
\text { puntuación de Sartorius del } \\
77,3 \text { y } 44,1 \% \text {, respectivamente } \\
\text { En ambos grupos se registró } \\
\text { una reducción significativa en } \\
\text { el HS-LASI después del } \\
\text { tratamiento, } 77,3 \pm 18,86 \% \text { en } \\
\text { los tratados con AM vs. } \\
44,1 \pm 28,19 \% \text { en los que se } \\
\text { aplicó gel libre de AM } \\
\text { No hubo diferencias respecto } \\
\text { a los efectos adversos (dolor, } \\
\text { eritema o hiperpigmentación) } \\
\text { ni correlación con el sitio } \\
\text { anatómico ni con el estadio de } \\
\text { la enfermedad } \\
\text { Efectos adversos: ninguno }\end{array}$ & $\begin{array}{l}\text { La combinación de AM como } \\
\text { fotosensibilizante activado } \\
\text { con IPL }(630 \mathrm{~nm} \text { ) es eficaz. El } \\
\text { uso de AM en niosomas } \\
\text { favorece la penetración del } \\
\text { fármaco en la dermis } \\
\text { Además atribuyen que la } \lambda \text { de } \\
630 \mathrm{~nm} \text { no solo fue una fuente } \\
\text { de activación de AM, sino } \\
\text { también un medio de la } \\
\text { destrucción del folículo piloso }\end{array}$ & $\begin{array}{l}\text { La limitación fue } \\
\text { el pequeño } \\
\text { tamaño muestral y } \\
\text { un periodo de } \\
\text { seguimiento corto. } \\
\text { Se requiere } \\
\text { realizar estudios } \\
\text { intraindividuo, } \\
\text { controlados, } \\
\text { aleatorizados, con } \\
\text { mayor tamaño de } \\
\text { muestra y periodos } \\
\text { de seguimiento } \\
\text { más largos, } \\
\text { además de } \\
\text { compararlos con } \\
\text { otros tratamientos } \\
\text { para la HS } \\
\text { (médicos y } \\
\text { quirúrgicos) }\end{array}$ & Media \\
\hline
\end{tabular}


Tabla 1 (continuación)

\begin{tabular}{|c|c|c|c|c|c|c|c|}
\hline Referencia & Estudio & Población & Intervención y seguimiento & Resultados & Conclusiones & Comentarios & $\begin{array}{l}\text { Calidad de la } \\
\text { evidencia }\end{array}$ \\
\hline $\begin{array}{l}\text { Ingram et al. }{ }^{3} \text {, } \\
2015\end{array}$ & $\begin{array}{l}\text { Diseño: revisión } \\
\text { sistemática, } \\
\text { estudios clínicos } \\
\text { aleatorizados } \\
\text { Objetivos: evaluar } \\
\text { los efectos de las } \\
\text { intervenciones } \\
\text { para HS en } \\
\text { personas de todas } \\
\text { las edades } \\
\text { Periodo de } \\
\text { búsqueda: } \\
\text { 1983-2015 }\end{array}$ & $\begin{array}{l}\text { Cualquier individuo, } \\
\text { incluyendo sexo, edad } \\
\text { y etnia, con un } \\
\text { diagnóstico clínico de } \\
\text { HS realizado por un } \\
\text { profesional médico, } \\
\text { basado en la definición } \\
\text { consensuada de la } \\
\text { enfermedad }\end{array}$ & 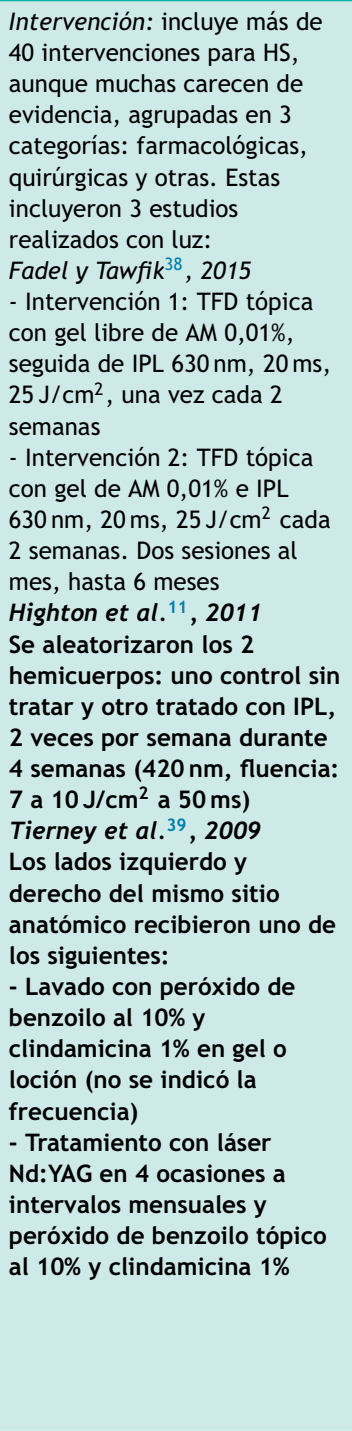 & $\begin{array}{l}\text { Número de estudios y pacientes: se } \\
\text { incluyeron } 12 \text { ensayos, con } 615 \\
\text { participantes. La mediana del número } \\
\text { de participantes fue } 27 \text { y la duración } \\
\text { media, de } 16 \text { semanas. Los estudios } \\
\text { incluidos se realizaron entre } \\
1983-2015 \\
\text { Magnitud del efecto: se asignó un } \\
\text { bajo grado a la calidad de la evidencia } \\
\text { de los } 3 \text { ensayos correspondientes a } \\
\text { las intervenciones con láser o luz } \\
\text { debido a imprecisión y falta de un } \\
\text { enmascaramiento adecuado } \\
\text { Fadel y Tawfik }{ }^{38}, 2015 \\
\text { TFD tópica con gel de AM } 0,01 \% \text { más } \\
\text { IPL fue superior a la TFD con gel de } \\
\text { AM libre } 0,01 \%, \text { con una reducción en } \\
\text { la puntuación de Sartorius del } 77,3 \text { y } \\
\text { el } 44,1 \%, \text { respectivamente } \\
\text { Highton et al. }{ }^{11,}, 2011 \\
\text { Beneficio significativo para IPL sin } \\
\text { compararla con otro tratamiento en } \\
18 \text { participantes, con una reducción } \\
\text { media de la puntuación Sartorius de } \\
12 \% \text { inmediatamente después de la } \\
\text { terapia, } 10 \% \text { a los } 3 \text { meses, } 10 \% \text { a los } \\
6 \text { y } 3 \% \text { a los } 12 \text {. La satisfacción con } \\
\text { el tratamiento se valoró con la } \\
\text { escala de Likert (sin validar) } \\
\text { Tierney et al. }{ }^{39}, 2009 \\
\text { Aplicó en } 17 \text { pacientes } 4 \\
\text { tratamientos de láser Nd: YAG en } \\
\text { intervalos mensuales. El tratamiento } \\
\text { fue administrado en un hemicuerpo, } \\
\text { en } 25 \text { regiones anatómicas con } \\
\text { lesiones de HS. Encontraron una } \\
\text { diferencia estadísticamente } \\
\text { significativa en la escala de Sartorius } \\
\text { modificada, en regiones axilares. } \\
\text { Reducción en la gravedad de la HS a } \\
\text { los } 3 \text { meses del } 65,3 \% \text { en todas las } \\
\text { localizaciones: } 73,4 \% \text { inguinal, } 62 \% \\
\text { axilar y } 53,1 \% \text { inframamaria }\end{array}$ & $\begin{array}{l}\text { Se califican de baja } \\
\text { calidad los estudios de } \\
\text { láser Nd:YAG, IPL y TFD } \\
\text { tópica debido a la } \\
\text { imprecisión, la falta de } \\
\text { enmascaramiento y la } \\
\text { ausencia de comparación } \\
\text { con placebo }\end{array}$ & & Alta \\
\hline
\end{tabular}


Tabla 1 (continuación)

\begin{tabular}{|c|c|c|c|c|c|c|c|}
\hline Referencia & Estudio & Población & Intervención y seguimiento & Resultados & Conclusiones & Comentarios & $\begin{array}{l}\text { Calidad de la } \\
\text { evidencia }\end{array}$ \\
\hline $\begin{array}{l}\text { John et al. } .^{52} \\
2016\end{array}$ & $\begin{array}{l}\text { Diseño: revisión } \\
\text { sistemática, } \\
\text { estudios clínicos } \\
\text { aleatorizados y no } \\
\text { aleatorizados, } \\
\text { revisiones } \\
\text { sistemáticas, } \\
\text { estudios de } \\
\text { cohortes de caso } \\
\text { control, series de } \\
\text { casos y opiniones } \\
\text { de expertos } \\
\text { Objetivos: evaluar } \\
\text { los efectos de las } \\
\text { intervenciones con } \\
\text { láser para HS en } \\
\text { personas de todas } \\
\text { las edades } \\
\text { Periodo de } \\
\text { búsqueda: } 1987 \text { a } \\
\text { abril del } 2016\end{array}$ & $\begin{array}{l}\text { Cualquier individuo, } \\
\text { incluyendo sexo, edad } \\
\text { y etnia, con un } \\
\text { diagnóstico clínico de } \\
\text { HS tratado con } \\
\text { cualquier tipo de láser }\end{array}$ & $\begin{array}{l}\text { Intervención: identificaron } 47 \\
\text { estudios pero finalmente } \\
\text { incluyeron según los criterios } \\
\text { de inclusión y exclusión } 22 \\
\text { publicaciones } \\
\text { Según la escala Jadad, solo } 2 \\
\text { estudios se graduaron como } \\
\text { un nivel tipo A y uno tipo B } \\
\text { Estas incluyeron } 3 \text { estudios } \\
\text { realizados con láser: } \\
\text { Con la mayor evidencia (A } \\
\text { Jadad), los ensayos de Highton } \\
\text { et al. de } 2011 \text { y Tierney et al. } \\
\text { previamente recogidos en la } \\
\text { revisión sistemática de Ingram } \\
\\
\text { Y además, con una } \\
\text { puntuación B de la escala } \\
\text { Jadad, el estudio prospectivo } \\
\text { de Xu et al. de } 2011 \\
X u \text { et al. }{ }^{45}, 2011 \text { realizaron } \\
\text { un estudio prospectivo } \\
\text { controlado en } 19 \text { pacientes } \\
\text { con HS grado II de Hurley } \\
\text { tratados con Nd:YAG con } \\
\text { longitud de onda de } \\
1.064 \text { nm que recibieron } 2 \\
\text { tratamientos mensuales }\end{array}$ & $\begin{array}{l}\text { Número de estudios y } \\
\text { pacientes: se incluyeron } 22 \\
\text { estudios. } 2 \text { con puntuación A y } \\
\text { uno con puntuación B; el } \\
\text { resto, con muy baja } \\
\text { evidencia, puntuación C } \\
\text { En total, los que tuvieron } \\
\text { evidencia A y B eran } 59 \\
\text { pacientes. Los estudios } \\
\text { incluidos se realizaron entre } \\
\text { 1987-2016 } \\
\text { Magnitud del efecto: se } \\
\text { asignó una calidad de la } \\
\text { evidencia A a los ensayos de } \\
\text { Highton et al. de } 2011 \text { y de } \\
\text { Tierney et al. de } 2009, \text { ya } \\
\text { explicado su efecto en la } \\
\text { revisión sistemática de Ingram } \\
\\
X u \text { et al. } 45,2011, \text { con } \\
\text { evidencia B, el porcentaje } \\
\text { de cambio en la puntuación } \\
\text { de Sartorius tras } 2 \text { meses de } \\
\text { tratamiento fue del } 31,6 \% \\
(p=0,001) \text { promediado para } \\
\text { todas las localizaciones } \\
\text { anatómicas, en región axilar } \\
\text { del } 24,4 \% \text { ( } \mathrm{p}=0,008 \text { y y del } \\
36,8 \% \text { para la región inguinal } \\
(\mathrm{p}=0,001) \text {. Tres pacientes, } \\
\text { sin embargo, continuaron } \\
\text { mostrando inflamación a } \\
\text { nivel histológico, y sus } \\
\text { porcentajes no mostraron } \\
\text { mejoría clínicamente } \\
\text { significativa }\end{array}$ & $\begin{array}{l}\text { Del análisis, los autores } \\
\text { extraen que hay evidencia } \\
\text { moderada para demostrar } \\
\text { la eficacia del láser } \\
\mathrm{Nd} \text { :YAG de pulso largo e } \\
\mathrm{IPL} \text { en el tratamiento de } \\
\mathrm{HS} \text { y la reducción del } \\
\text { riesgo de recurrencia, a } \\
\text { través del mecanismo de } \\
\text { depilación, y evidencia } \\
\text { limitada del láser de } \mathrm{CO}_{2}\end{array}$ & $\begin{array}{l}\text { Destacan la necesidad } \\
\text { de estudios } \\
\text { aleatorizados de gran } \\
\text { tamaño, } \\
\text { preferiblemente } \\
\text { multicéntricos, para la } \\
\text { estandarización de las } \\
\text { técnicas de evaluación } \\
\text { y valorar la eficacia }\end{array}$ & Alta \\
\hline
\end{tabular}




\begin{tabular}{|c|c|c|c|c|c|c|c|}
\hline Referencia & Estudio & Población & Intervención y seguimiento & Resultados & Conclusiones & Comentarios & $\begin{array}{l}\text { Calidad de la } \\
\text { evidencia }\end{array}$ \\
\hline $\begin{array}{l}\text { Robert et al. }{ }^{53} \text {, } \\
2017\end{array}$ & $\begin{array}{l}\text { Diseño: revisión } \\
\text { sistemática, } \\
\text { estudios clínicos } \\
\text { aleatorizados y no } \\
\text { aleatorizados, } \\
\text { revisiones } \\
\text { sistemáticas, } \\
\text { estudios de } \\
\text { cohortes de caso } \\
\text { control, series de } \\
\text { casos y opiniones } \\
\text { de expertos } \\
\text { Objetivos: realizar } \\
\text { un análisis } \\
\text { sintético de los } \\
\text { procedimientos no } \\
\text { quirúrgicos } \\
\text { actualmente } \\
\text { disponibles en la } \\
\text { HS } \\
\text { Periodo de } \\
\text { búsqueda: enero } \\
\text { del } 2005 \text { a } \\
\text { septiembre del } \\
2015\end{array}$ & $\begin{array}{l}\text { Se incluyeron } \\
\text { todos los artículos } \\
\text { originales } \\
\text { relacionados con } \\
\text { tratamiento no } \\
\text { quirúrgico de la HS } \\
\text { (ensayos } \\
\text { controlados y } \\
\text { observacionales } \\
\text { prospectivo o } \\
\text { retrospectivo y } \\
\text { series de casos de } \\
\text { al menos } 4 \\
\text { pacientes) } \\
\text { Se excluyeron: } \\
\text { casos clínicos } \\
\text { aislados, } \\
\text { revisiones de la } \\
\text { literatura, } \\
\text { discusiones, } \\
\text { comentarios, } \\
\text { cartas científicas y } \\
\text { editoriales }\end{array}$ & 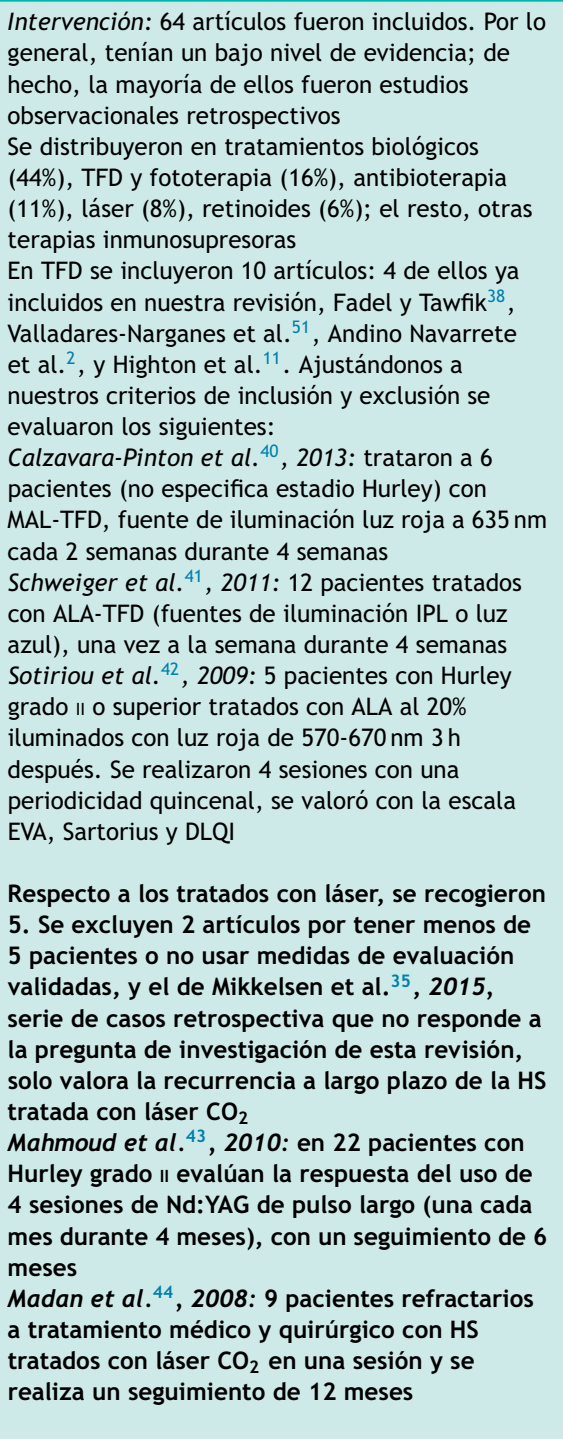 & 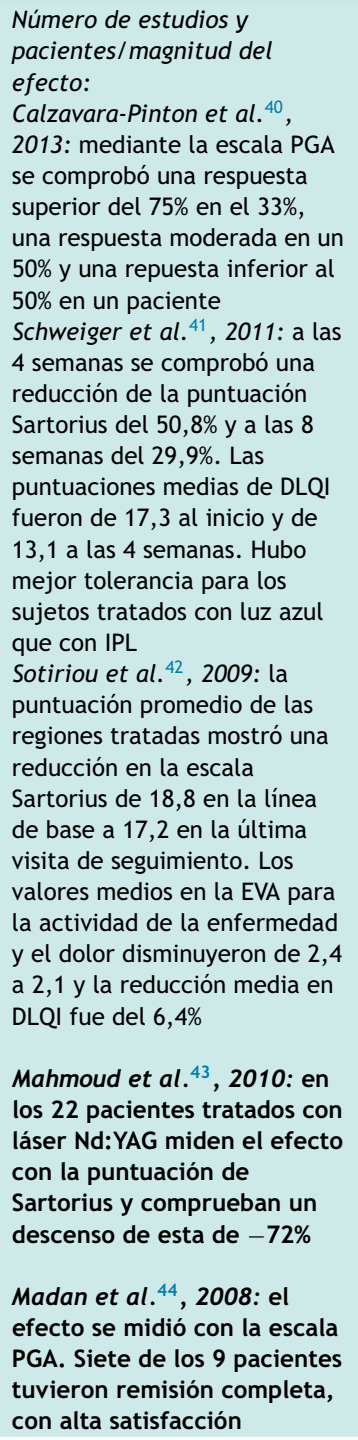 & $\begin{array}{l}\text { Del análisis, los } \\
\text { autores extraen que } \\
\text { ningún tratamiento no } \\
\text { quirúrgico provoca una } \\
\text { remisión a largo plazo } \\
\text { Destacan el } \\
\text { tratamiento quirúrgico } \\
\text { en las formas } \\
\text { recalcitrantes y } \\
\text { debilitantes }\end{array}$ & $\begin{array}{l}\text { Estudios de } \\
\text { baja calidad de } \\
\text { evidencia. Los } \\
\text { métodos de } \\
\text { evaluación son } \\
\text { heterogéneos y } \\
\text { apenas } \\
\text { comparables. } \\
\text { Existen pocos } \\
\text { estudios } \\
\text { controlados } \\
\text { aleatorios, y la } \\
\text { mayoría de los } \\
\text { estudios de } \\
\text { cohortes } \\
\text { recogidos son } \\
\text { retrospectivos }\end{array}$ & Alta \\
\hline
\end{tabular}

ALA: ácido 5-aminolevulínico; AM: azul de metileno; DLQI: índice de calidad de vida dermatológica; EVA: escala visual analógica; HS: hidrosadenitis supurativa; HS-LASI: Índice de Lesión, Área y Gravedad de la Hidrosadenitis supurativa; IPL: luz pulsada intensa; MAL: metilaminolevulinato; Nd:YAG: neodymium-doped yttrium aluminium garnet; TFD: terapia fotodinámica.

En negrita están resaltados artículos recogidos en las revisiones sistemáticas en los que la fuente de luz fue diferente a la TFD. 
Tabla 2 Resumen de los artículos en los que se trata la hidrosadenitis supurativa con terapia fotodinámica intralesional

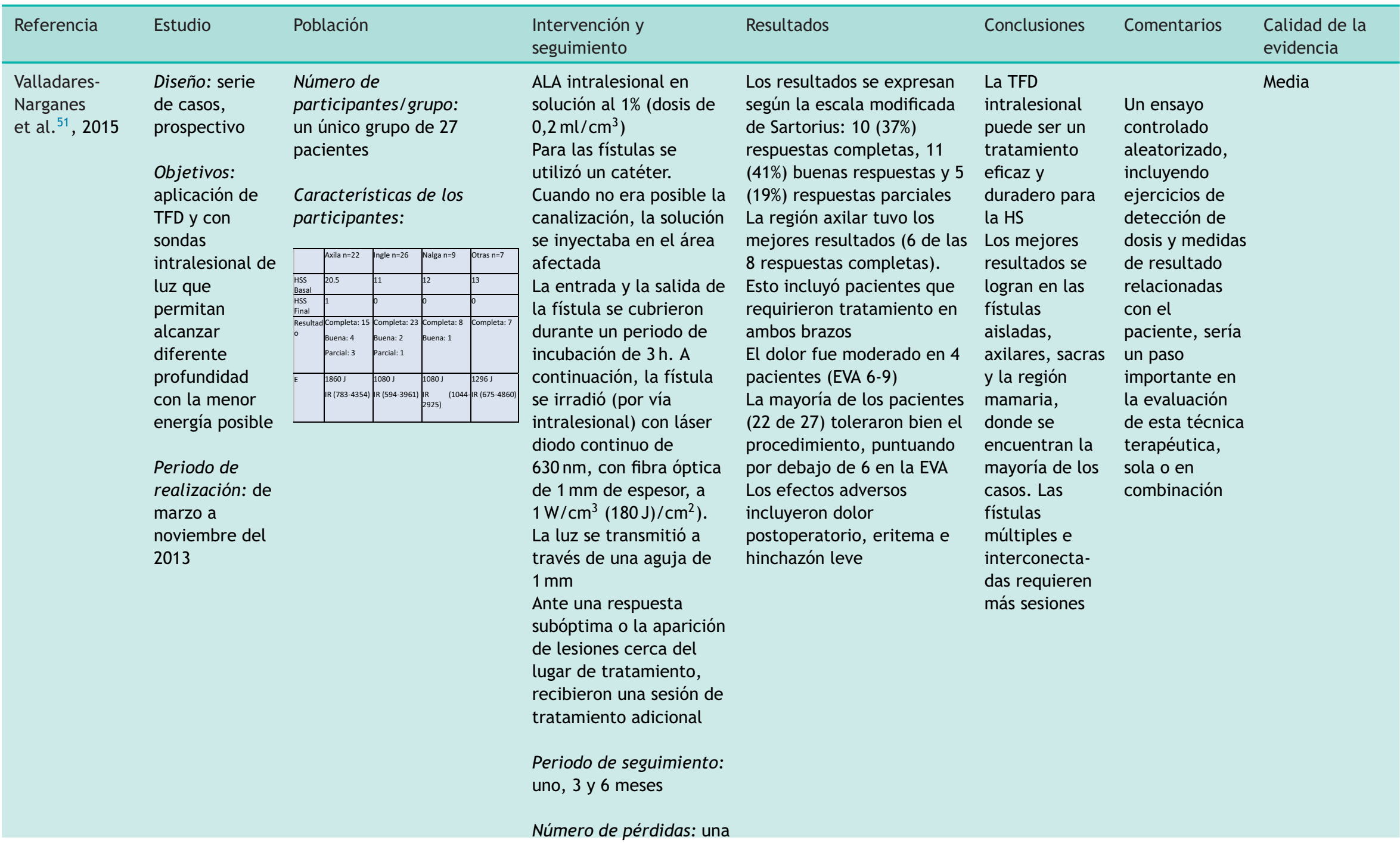


Tabla 2 (continuación)

\begin{tabular}{|c|c|c|c|c|c|c|c|}
\hline Referencia & Estudio & Población & $\begin{array}{l}\text { Intervención y } \\
\text { seguimiento }\end{array}$ & Resultados & Conclusiones & Comentarios & $\begin{array}{l}\text { Calidad de la } \\
\text { evidencia }\end{array}$ \\
\hline $\begin{array}{l}\text { Agut-Busquet } \\
\text { et al. }{ }^{1}, 2016\end{array}$ & $\begin{array}{l}\text { Diseño: serie } \\
\text { de casos, } \\
\text { retrospectivo } \\
\text { Objetivos: } \\
\text { comunicar la } \\
\text { experiencia en } \\
7 \text { pacientes con } \\
\text { HS, tratados } \\
\text { con TFD, } \\
\text { aplicando AM } \\
\text { intralesional y } \\
\text { luz } 635 \text { nm } \\
\text { Periodo de } \\
\text { realización: } 8 \\
\text { meses }\end{array}$ & $\begin{array}{l}\text { Número de } \\
\text { participantes/grupo: un } \\
\text { grupo de } 7 \text { pacientes } \\
\text { Características de los } \\
\text { participantes: pacientes } \\
\text { con HS, en estadio clínico ॥ } \\
\text { y II de Hurley, con al menos } \\
\text { una zona activa }\end{array}$ & $\begin{array}{l}\text { Solución intralesional de } \\
\text { AM } 1 \% \text {, inyectada con } \\
\text { guía ecográfica o sin ella } \\
\text { Tras incubación de } \\
15 \text { min, iluminación con } \\
\text { lámpara LED de } 635 \mathrm{~nm} \\
\left(37 \mathrm{~J} / \mathrm{cm}^{2}\right) \text {. } \\
1-2 \text { sesiones separadas } \\
15 \text { días } \\
\text { Periodo de seguimiento: } \\
1,2,4 \text { y } 6 \text { meses } \\
\text { Número de pérdidas: una }\end{array}$ & $\begin{array}{l}\text { Pacientes con Hurley grado } \\
\text { II respondieron mejor que } \\
\text { aquellos con grado III ( } 99 \\
\text { versus } 87 \% \text { de mejora, } \\
\text { respectivamente) y la } \\
\text { región inguinal tuvo los } \\
\text { mejores resultados } \\
\text { Mejoría del DQLI en } 6 \\
\text { pacientes: uno del } 78 \% \text {, otro } \\
\text { del } 80 \%, 2 \text { del } 90 \% \text { y } 2 \text { del } \\
99 \% \\
\text { Se valoró la respuesta } \\
\text { mediante la escala Sartorius } \\
\text { y PGA: en uno de ellos la } \\
\text { respuesta fue del } 78 \%, \text { en } \\
\text { otro paciente del } 80 \%, 2 \\
\text { pacientes con un } 90 \% \text { y otros } \\
2 \text { con un } 99 \% \text { de respuesta } \\
\text { Únicamente en un paciente } \\
\text { se objetivó una buena } \\
\text { respuesta (86\%) después del } \\
\text { tratamiento con } \\
\text { antibióticos } \\
\text { Efectos adversos: leves en } 6 \\
\text { casos (86\%). Un paciente } \\
\text { presentó un evento adverso } \\
\text { grave: celulitis en el sitio } \\
\text { tratado, que se atribuyó a la } \\
\text { automanipulación después } \\
\text { de la sesión de terapia }\end{array}$ & $\begin{array}{l}\text { La TFD con AM } \\
\text { intralesional } \\
\text { tiene buenos } \\
\text { resultados en } \\
\text { HS. Más eficaz } \\
\text { en Hurley } \\
\text { grado ॥ que III } \\
\text { Se necesitan } \\
\text { ensayos } \\
\text { clínicos para } \\
\text { aumentar la } \\
\text { evidencia }\end{array}$ & $\begin{array}{l}\text { Escaso número } \\
\text { de pacientes, } \\
\text { lo que dificulta } \\
\text { la } \\
\text { extrapolación a } \\
\text { la población } \\
\text { general } \\
\text { (validez } \\
\text { externa) }\end{array}$ & Baja \\
\hline
\end{tabular}


Tabla 2 (continuación)

\begin{tabular}{|c|c|c|c|c|c|c|c|}
\hline Referencia & Estudio & Población & $\begin{array}{l}\text { Intervención y } \\
\text { seguimiento }\end{array}$ & Resultados & Conclusiones & Comentarios & $\begin{array}{l}\text { Calidad de la } \\
\text { evidencia }\end{array}$ \\
\hline $\begin{array}{l}\text { Suárez } \\
\text { Valladares } \\
\text { et al. }{ }^{50}, 2017\end{array}$ & $\begin{array}{l}\text { Diseño: serie } \\
\text { de casos, } \\
\text { prospectivo } \\
\text { Objetivos: } \\
\text { evaluar la } \\
\text { efectividad de } \\
\text { la TFD } \\
\text { intralesional en } \\
\text { el manejo local } \\
\text { de la HS a } \\
\text { corto y medio } \\
\text { plazo, en las } \\
\text { diferentes } \\
\text { áreas } \\
\text { anatómicas } \\
\text { Periodo de } \\
\text { realización: } \\
\text { marzo del } \\
2011 \text { - } \\
\text { noviembre del } \\
2015\end{array}$ & $\begin{array}{l}\text { Número de } \\
\text { participantes/grupo: un } \\
\text { solo grupo de } 38 \text { pacientes } \\
\text { Características de los } \\
\text { participantes: } \\
\text { Sexo: } 20 \text { mujeres y } 18 \\
\text { hombres } \\
\text { Edad: } 30-44 \text { años } \\
\text { Peso: } 67,25-90 \mathrm{~kg} \\
\text { IMC: } 26,87 \pm 4,89 \\
\text { IMC mayor de } 27: 20 \\
\text { Fumador: } 34 \\
\text { Tratamientos previos: } \\
\text { ningún tratamiento } 2 \text {, } \\
\text { tópico } 1, \text { antibióticos orales } \\
23 \text {, retinoides orales } 2 \text {, } \\
\text { biológicos } 1 \text {, cirugía } 9 \text {. } \\
\text { Estadio de Hurley: grado I, } 4 \\
\text { pacientes; II, } 21 \text { pacientes; } \\
\text { III, } 13 \text { pacientes } \\
\text { Las medidas directas de } \\
\text { gravedad (Hurley, valor } \\
\text { basal del HSS total y el DLQI } \\
\text { también mostraron una alta } \\
\text { gravedad de la enfermedad }\end{array}$ & $\begin{array}{l}\text { Tras administrar } \\
\text { anestesia local, se } \\
\text { canalizó el ALA 5\% gel a } \\
\text { través de una cánula de } \\
\text { plástico, llenando los } \\
\text { trayectos fistulosos. Así, } \\
\text { las paredes de la fístula } \\
\text { se tratan } \\
\text { independientemente de } \\
\text { la profundidad, sin dejar } \\
\text { áreas no tratadas. En los } \\
\text { nódulos se inyectó una } \\
\text { solución liofilizada al } 1 \% \text {. } \\
\text { Las lesiones se incubaron } \\
\text { bajo oclusión opaca } \\
\text { durante } 2 \mathrm{~h} \text { antes de ser } \\
\text { irradiadas. La irradiación } \\
(1,2 \mathrm{~W} \text { y la fluencia } \\
\text { (180 J/cm²) se hizo con } \\
\text { láser intralesional de } \\
630 \mathrm{~nm} \text {, con sonda de } \\
\text { fibra óptica de } 400 \text { mm } \\
\text { insertada dentro de una } \\
\text { aguja } \\
\text { Seguimiento cada } 5-7 \\
\text { semanas } \\
\text { Periodo de seguimiento: } \\
26,21 \text { ( } 21,07-68,57 \text { ) } \\
\text { meses } \\
\text { Número de pérdidas: } \\
\text { cero }\end{array}$ & $\begin{array}{l}\text { El número medio de } \\
\text { sesiones fue una, sin diferir } \\
\text { entre las distintas zonas } \\
\text { corporales, pero la mediana } \\
\text { de la energía administrada } \\
\text { fue mayor en el área axilar } \\
\text { Se observó remisión } \\
\text { completa en el } 76,3 \% \text { de los } \\
\text { pacientes tratados, con un } \\
\text { intervalo medio libre de } \\
\text { enfermedad de } 168 \\
\text { semanas. La aparición de } \\
\text { nuevas lesiones en áreas no } \\
\text { tratadas se observó en } 9 \\
\text { pacientes ( } 23,7 \% \text { ), de los } \\
\text { cuales } 6 \text { alcanzaron la } \\
\text { remisión completa } \\
\text { Nueve pacientes no } \\
\text { alcanzaron la remisión o } \\
\text { presentaron recurrencia } \\
\text { tras el tratamiento. Estos } \\
\text { presentaron un porcentaje } \\
\text { inferior de obesidad ( } 33,3 \\
\text { vs. } 58,6 \% \text { y tabaquismo } \\
(66,7 \text { vs. } 96,6 \% \text { ), pero una } \\
\text { enfermedad inicial más } \\
\text { grave ( } 44,4 \text { vs. } 31 \% \text { y una } \\
\text { puntuación basal en la } \\
\text { escala de Sartorius } \\
\text { modificada ( } 36 \text { vs. } 22 \text { ) y de } \\
\text { DLQl ( } 16 \text { vs. } 10 \text { ) inferiores a } \\
\text { las de aquellos en los que la } \\
\text { TFD fue efectiva }\end{array}$ & $\begin{array}{l}\text { La TFD } \\
\text { intralesional } \\
\text { podría ser una } \\
\text { nueva } \\
\text { alternativa } \\
\text { para tratar las } \\
\text { lesiones de la } \\
\text { HS, ya sea en } \\
\text { monoterapia o } \\
\text { adyuvante } \\
\text { Logra alta tasa } \\
\text { de remisión } \\
\text { completa, con } \\
\text { un } \\
\text { mantenimiento } \\
\text { prolongado } \\
\text { No se han } \\
\text { observado } \\
\text { complicaciones } \\
\text { Rápida } \\
\text { recuperación } \\
\text { tras el } \\
\text { tratamiento y } \\
\text { posibilidad de } \\
\text { repetir } \\
\text { sesiones según } \\
\text { sea necesario }\end{array}$ & & Media \\
\hline
\end{tabular}

AM: azul de metileno; DLQI: índice de calidad de vida dermatológica; E: energía; EVA: escala visual analógica; HS: hidradenitis supurativa; IMC: índice de masa corporal; PGA: escala de evaluación global del médico; TFD: terapia fotodinámica. 
Tabla 3 Resumen de los artículos de los diferentes tipos de luz en hidrosadenitis supurativa incluidos en la revisión sistemática

\begin{tabular}{|c|c|c|c|c|c|c|c|}
\hline Referencia & Estudio & Población & $\begin{array}{l}\text { Intervención y } \\
\text { seguimiento }\end{array}$ & Resultados & Conclusiones & Comentarios & $\begin{array}{l}\text { Calidad de la } \\
\text { evidencia }\end{array}$ \\
\hline \multicolumn{8}{|l|}{$\mathrm{Nd}: Y A G$} \\
\hline $\begin{array}{l}\text { Zerbinati } \\
\text { et al. }{ }^{47}, 2017\end{array}$ & $\begin{array}{l}\text { Diseño: serie de } \\
\text { casos, prospectivo } \\
\text { Objetivos: evaluar } \\
\text { los efectos clínicos } \\
\text { e histológicos en } \\
\text { pacientes con HS } \\
\text { leve tratada con } \\
\text { láser Nd:YAG } \\
\text { Periodo de } \\
\text { realización: no lo } \\
\text { especifica }\end{array}$ & $\begin{array}{l}\text { Número de parti- } \\
\text { cipantes/grupo: } \\
12 \text { pacientes } \\
\text { Características de } \\
\text { los participantes: } \\
\text { pacientes con HS } \\
\text { tipo I } \\
\text { Edad media } 39,5 \\
\text { años (rango: } 24 \text { a } \\
50 \text { años). Con } \\
\text { afectación } \\
\text { bilateral de la } \\
\text { enfermedad }\end{array}$ & $\begin{array}{l}\text { Se utilizó láser } \\
\text { Nd:YAG con una } \\
\text { longitud de onda } \\
\text { de } 1.064 \mathrm{~nm} \text {, en } \\
\text { fibra de } \\
300-600 \mu \mathrm{m} \text { (fibra } \\
\text { de vidrio } \\
\text { recubierta de } \\
\text { policarbonato) } \\
\text { Fluencia de } \\
300 \mathrm{~J} / \mathrm{cm}^{2} \text {, } \\
\text { empleando } \\
\text { diferentes } \\
\text { diámetros de } \\
\text { fibras según el tipo } \\
\text { de lesión y su } \\
\text { localización } \\
\text { anatómica } \\
\text { La energía del } \\
\text { pulso fue de } 150 \text { a } \\
350 \text { mJ } \\
\text { Se realizaron } 3 \\
\text { sesiones de láser, } \\
\text { una cada } 2 \text { meses } \\
\text { Periodo de } \\
\text { seguimiento: } 12 \\
\text { meses } \\
\text { Número de } \\
\text { pérdidas: cero }\end{array}$ & $\begin{array}{l}\text { Una media de } \\
\text { mejoría en el } \\
\text { porcentaje de } \\
\text { Sartorius del } 85,3 \% \\
\text { Histológicamente } \\
\text { se comprobó un } \\
\text { descenso del } \\
\text { infiltrado } \\
\text { inflamatorio, } \\
\text { seguido de una } \\
\text { fibrosis } 4 \text { meses } \\
\text { después del } \\
\text { tratamiento }\end{array}$ & $\begin{array}{l}\text { El láser Nd:YAG se } \\
\text { trata de una } \\
\text { técnica no invasiva } \\
\text { bien tolerada que } \\
\text { es una alternativa } \\
\text { terapéutica en } \\
\text { esta enfermedad } \\
\text { crónica y } \\
\text { debilitante }\end{array}$ & $\begin{array}{l}\text { Se precisa un } \\
\text { mayor número de } \\
\text { pacientes y un } \\
\text { seguimiento más } \\
\text { amplio para sacar } \\
\text { conclusiones con } \\
\text { mayor evidencia }\end{array}$ & Baja \\
\hline
\end{tabular}


Tabla 3 (continuación)

\begin{tabular}{|c|c|c|c|c|c|c|c|}
\hline Referencia & Estudio & Población & $\begin{array}{l}\text { Intervención y } \\
\text { seguimiento }\end{array}$ & Resultados & Conclusiones & Comentarios & $\begin{array}{l}\text { Calidad de } \\
\text { la evidencia }\end{array}$ \\
\hline \multicolumn{8}{|l|}{$\mathrm{Nd}: Y A G$ y $\mathrm{CO}_{2}$} \\
\hline $\begin{array}{l}\text { Abdel Azim } \\
\text { et al. }{ }^{48}, 2018\end{array}$ & $\begin{array}{l}\text { Diseño: ensayo } \\
\text { clínico } \\
\text { aleatorizado, } \\
\text { ciego } \\
\text { intraindividual } \\
\text { Objetivos: } \\
\text { estudiar la } \\
\text { seguridad y la } \\
\text { eficacia del } \\
\text { tratamiento } \\
\text { combinado con } \\
\text { láser fraccional } \\
\mathrm{CO}_{2} \text { y láser } \\
\text { Nd:YAG de pulso } \\
\text { largo (1.064 nm) } \\
\text { en el tratamiento } \\
\text { de la HS } \\
\text { Periodo de } \\
\text { realización: de } \\
\text { enero a diciembre } \\
\text { del } 2017\end{array}$ & $\begin{array}{l}\text { Número de parti- } \\
\text { cipantes/grupo: se } \\
\text { inscribieron } 30 \text { y } \\
\text { se excluyeron } 10 \\
\text { (finalmente hubo } \\
20 \text { pacientes) } \\
\text { Características de } \\
\text { los participantes: } \\
\text { pacientes mayores } \\
\text { de } 18 \text { años con HS } \\
\text { grado I o ॥ de } \\
\text { Hurley con } \\
\text { lesiones } \\
\text { bilaterales }\end{array}$ & $\begin{array}{l}\text { Intervención grupo } \\
\text { experimental: los } \\
\text { pacientes } \\
\text { recibieron } 4 \\
\text { sesiones de láser } \\
\text { con un intervalo } \\
\text { de } 2 \text { semanas. El } \\
\text { lado del } \\
\text { tratamiento } \\
\text { combinado recibió } \\
\text { láser de CO } 2 \\
\text { fraccionado } \\
\text { seguido de Nd:YAG } \\
\text { de pulso largo } \\
\text { (1.064 nm) en la } \\
\text { misma sesión } \\
\text { Intervención grupo } \\
\text { control: control } \\
\text { intrapaciente un } \\
\text { lado (lado de } \\
\text { control) recibió } \\
\text { Nd:YAG de pulso } \\
\text { largo (1.064 nm) } \\
\text { Periodo de } \\
\text { seguimiento: } 3 \\
\text { meses tras la } \\
\text { última sesión } \\
\text { Pérdidas postalea- } \\
\text { torización: } 5 \text { (en } \\
\text { el seguimiento) } \\
\text { para evaluar el } \\
\text { mantenimiento de } \\
\text { la respuesta }\end{array}$ & $\begin{array}{l}\text { Se observó mejoría } \\
\text { estadísticamente significativa y } \\
\text { mayor satisfacción del paciente } \\
\text { en el lado del tratamiento } \\
\text { combinado en comparación con el } \\
\text { lado de control ( } \mathrm{p}=0,011 \text { y } 0,048 \text {, } \\
\text { respectivamente). Se valoró } \\
\text { mediante la escala PGA la } \\
\text { respuesta al tratamiento, } \\
\text { demostrándose una ausencia de } \\
\text { recurrencia en el } 55 \% \text { de los lados } \\
\text { que recibieron tratamiento } \\
\text { combinado y el } 35 \% \text { de los lados } \\
\text { de control. El examen } \\
\text { histopatológico de piel a las } 2 \\
\text { semanas de la última sesión reveló } \\
\text { escaso infiltrado linfohistiocítico } \\
\text { perifolicular para los lados del } \\
\text { tratamiento combinado. Por otro } \\
\text { lado, hubo una menor reducción } \\
\text { del infiltrado perifolicular y del } \\
\text { edema dérmico para lados Nd:YAG } \\
\text { en comparación con lados del } \\
\text { tratamiento combinado. No se } \\
\text { informaron efectos secundarios, a } \\
\text { excepción del eritema, que se } \\
\text { resolvió espontáneamente dentro } \\
\text { de las } 48 \text { h posteriores al } \\
\text { tratamiento } \\
\text { Efectos adversos: solo en un } \\
\text { paciente }\end{array}$ & $\begin{array}{l}\text { La combinación de } \\
\text { láser } \mathrm{CO}_{2} \\
\text { fraccionado y láser } \\
\text { de pulso largo } \\
\text { Nd:YAG ( } 1.064 \mathrm{~nm}) \\
\text { consigue un mayor } \\
\text { grado de mejoría y } \\
\text { satisfacción del } \\
\text { paciente junto con } \\
\text { una menor } \\
\text { recurrencia en } \\
\text { comparación con } \\
\text { Nd:YAG de pulso } \\
\text { largo (1.064 nm) } \\
\text { en monoterapia } \\
\text { para la HS de las } \\
\text { etapas I y ıde } \\
\text { Hurley. Se podrían } \\
\text { lograr mejores } \\
\text { resultados en } \\
\text { pacientes con HS } \\
\text { con una } \\
\text { puntuación baja } \\
\text { de PGA, pacientes } \\
\text { no obesos, y la } \\
\text { ausencia de } \\
\text { cirugía previa en } \\
\text { el sitio de la lesión }\end{array}$ & $\begin{array}{l}\text { La limitación } \\
\text { de este estudio } \\
\text { fue un tamaño } \\
\text { muestral } \\
\text { pequeño y un } \\
\text { periodo de } \\
\text { seguimiento } \\
\text { corto. Se } \\
\text { requiere } \\
\text { analizar } \\
\text { estudios } \\
\text { intraindividua- } \\
\text { les controlados } \\
\text { aleatorizados } \\
\text { con mayor } \\
\text { tamaño de } \\
\text { muestra y } \\
\text { periodos de } \\
\text { seguimiento } \\
\text { más largos, } \\
\text { además de } \\
\text { compararlos } \\
\text { con } \\
\text { tratamiento } \\
\text { médico y } \\
\text { quirúrgico de la } \\
\text { HS }\end{array}$ & Media \\
\hline
\end{tabular}


Tabla 3 (continuación)

\begin{tabular}{|c|c|c|c|c|c|c|c|}
\hline Referencia & Estudio & Población & Intervención y seguimiento & Resultados & Conclusiones & Comentarios & $\begin{array}{l}\text { Calidad de } \\
\text { la evidencia }\end{array}$ \\
\hline \multicolumn{8}{|c|}{ Láser diodo intralesional } \\
\hline $\begin{array}{l}\text { Fabbrocini } \\
\text { et al. }{ }^{49}, 2018\end{array}$ & $\begin{array}{l}\text { Diseño: serie de } \\
\text { casos, prospectivo } \\
\text { Objetivos: evaluar } \\
\text { la eficacia del } \\
\text { láser diodo de } \\
1.064 \mathrm{~nm} \\
\text { intralesional en HS } \\
\text { leve-moderada } \\
\text { Periodo de } \\
\text { realización: de } \\
\text { mayo del } 2016 \text { a } \\
\text { enero del } 2017\end{array}$ & $\begin{array}{l}\text { Número de parti- } \\
\text { cipantes/grupo: } \\
\text { un grupo de } 20 \\
\text { pacientes } \\
\text { Características de } \\
\text { los participantes: } \\
\text { pacientes con HS } \\
\text { Hurley estadio I-II, } \\
\text { PGA } \\
\text { leve-moderado }\end{array}$ & $\begin{array}{l}\text { Se utilizó láser de diodo con } \\
\text { una longitud de onda de } \\
1.064 \mathrm{~nm} \text {,en fibra de } \\
300-600 \mu \mathrm{m} \text { (fibra de vidrio } \\
\text { recubierta de } \\
\text { policarbonato) } \\
\text { Fluencia de } 250 \mathrm{~J} / \mathrm{cm}^{2} \text {, } \\
\text { empleando diferentes } \\
\text { diámetros de fibras según el } \\
\text { tipo de lesión y su } \\
\text { localización anatómica } \\
\text { La energía del pulso fue de } \\
150 \text { a } 350 \text { ms y la potencia } \\
\text { de energía seleccionada, de } \\
6-8-10 \text { W. Se usó una aguja } \\
\text { de } 30 \mathrm{G} \text { y se administró } \\
\text { aproximadamente } 1 \text { ml de } \\
\text { solución anestésica por } \\
\text { cm². La infiltración se } \\
\text { practicó hasta obtener la } \\
\text { tumescencia del área } \\
\text { afectada } \\
\text { Desde el día del } \\
\text { procedimiento del láser } \\
\text { quirúrgico, todos los } \\
\text { pacientes fueron sometidos } \\
\text { a un tratamiento antibiótico } \\
\text { oral con } 500 \text { mg de } \\
\text { azitromicina una vez al día } \\
\text { durante } 3 \text { días } \\
\text { Se realizaron } 4 \text { sesiones de } \\
\text { tratamiento, una cada } 2 \\
\text { semanas }\end{array}$ & $\begin{array}{l}\text { Cada paciente se } \\
\text { sometió a } 4 \text { sesiones de } \\
\text { láser, una cada } 2 \\
\text { semanas. Se registró una } \\
\text { reducción significativa } \\
\text { (31\%) de la puntuación } \\
\text { de Sartorius de } \\
28,55 \pm 13,04 \text { a } \\
19,75 \pm 12,29 \text { después de } \\
4 \text { sesiones de láser } \\
\text { ( } p<0,05) \text {. Nadie ha } \\
\text { empeorado } \\
\text { En paralelo con la } \\
\text { mejoría clínica, se } \\
\text { notificó una mejora } \\
\text { notable en la calidad de } \\
\text { vida de los pacientes } \\
\text { sometidos al } \\
\text { tratamiento, demostrada } \\
\text { por la reducción de los } \\
\text { valores de DLQl } \\
\text { ( } p=0,0307 \text { ) }\end{array}$ & $\begin{array}{l}\text { El láser de diodo } \\
\text { intralesional } \\
1.064 \text { nm puede } \\
\text { ser una buena } \\
\text { opción de } \\
\text { tratamiento para } \\
\text { pacientes con HS } \\
\text { moderada y } \\
\text { localizada, ya que } \\
\text { es mínimamente } \\
\text { invasiva, no } \\
\text { presenta } \\
\text { complicaciones } \\
\text { significativas y } \\
\text { proporciona una } \\
\text { recuperación } \\
\text { postratamiento } \\
\text { rápida }\end{array}$ & $\begin{array}{l}\text { Escasos } \\
\text { pacientes, } \\
\text { evidencia baja }\end{array}$ & Baja \\
\hline
\end{tabular}

DLQI: índice de calidad de vida dermatológica; HS: hidradenitis supurativa; Nd:YAG: neodymium-doped yttrium aluminium garnet; PGA: escala de evaluación global del médico. 
no validada y Madan et al. ${ }^{44}$ mediante una puntuación de 0 al 10 (siendo 0 muy insatisfecho y 10 muy satisfecho).

Dos de los estudios aportan datos sobre el dolor presentado durante y después del tratamiento, valorado mediante la escala visual analógica ${ }^{2,51}$.

En cuanto al informe de efectos adversos, son 4 los trabajos que hablan sobre ello ${ }^{1,2,51}$, si bien Highton et al. ${ }^{11}$ solo informan de efectos adversos que condujeran a la interrupción del tratamiento.

Por último, 5 estudios dan la recurrencia de enfermedad después del tratamiento: Agut-Busquet et al. ${ }^{1}$ destacan la recidiva explicando la evolución de cada uno de los pacientes después del tratamiento; Suárez Valladares et al. ${ }^{50}$ señalan recurrencia en 9 de sus pacientes; Highton et al. ${ }^{11}$ indican una posible tendencia a la recidiva a los 12 meses del tratamiento. Abdel Azim et al. ${ }^{48}$ también valoran la recurrencia a los 3 meses y Madan et al. ${ }^{44}$ a los 12 meses tras el tratamiento.

\section{Resultados de las distintas modalidades de terapia con luz}

Los resultados, así como la metodología y la calidad de la evidencia, se representan en las tablas 1-3, divididas en HS tratadas con TFD convencional, con TFD intralesional y con láser. Como ya hemos comentado, en las 3 revisiones sistemáticas se engloban pacientes tratados con TFD y con láser; con el fin de evitar duplicidades, se representan en una sola tabla (tabla 1), resaltando los estudios en los que el tratamiento fue láser o IPL.

\section{Terapia fotodinámica tópica}

Andino Navarrete et al. ${ }^{2}$ aplicaron en 5 pacientes una solución de ALA al $20 \%$ durante $1,5 \mathrm{~h}$, irradiando posteriormente con lámpara LED de $635 \mathrm{~nm}$, fluencia de $37 \mathrm{~J} / \mathrm{cm}^{2}$ e intensidad de $70 \mathrm{~mW} / \mathrm{cm}^{2}$ por sesión. Los pacientes mostraron una mejoría significativa en la escala de Sartorius, el DLQI y la escala visual analógica, mantenida a las 8 semanas de seguimiento, aunque los resultados no fueron estadísticamente significativos.

Fadel y Tawfik ${ }^{38}$ comparan la aplicación de TFD con un gel de AM al 0,01\% tópico (formulación con liposomas basada en surfactantes para incrementar la penetración del fotosensibilizante), o un gel libre de $A M$, seguidos de irradiación con IPL de $630 \mathrm{~nm}$, durante $20 \mathrm{~ms}$ administrando $25 \mathrm{~J} / \mathrm{cm}^{2}$. Los participantes fueron aleatorizados para recibir una intervención en cada parte del cuerpo, tratando axilas, nalgas, ingles y región inframamaria. Los resultados mostraron una reducción en la escala de Sartorius del 77,3 y el $44,1 \%$ con el gel de AM liposomal y el gel libre de AM, respectivamente $(p<0,01)$, por lo que se concluyen que la TFD con $A M$ en liposomas es superior a la TFD con AM libre ${ }^{38}$.

Calzavara-Pinton et al. ${ }^{40}$, en un estudio que recoge diferentes enfermedades tratadas con MAL-TFD, recopilan 6 casos de hidradenitis supurativa tratados con MAL-TFD con fuente de iluminación de luz roja a $635 \mathrm{~nm}$ cada 2 semanas durante 4 semanas, demostrando una respuesta en la PGA superior al $75 \%$ en el $33 \%$ de los casos, una respuesta moderada en un $50 \%$ y una repuesta inferior al $50 \%$ en un paciente.
Schweiger et al. ${ }^{41}$ reportan 12 pacientes tratados con ALA-TFD con fuentes de iluminación con IPL o luz azul, una vez a la semana durante 4 semanas. A las 4 semanas se comprobó una reducción de la puntuación Sartorius del 50,8\% y a las 8 semanas del $29,9 \%$, con una reducción del DLQI de 17,3 a 13,1, con mejor tolerancia con luz azul que con IPL.

Por último, Sotiriou et al. ${ }^{42}$ usaron ALA-TFD al $20 \%$ en 5 pacientes con Hurley grado ॥ o superior, administrando 5 sesiones. Como resultados obtuvieron una reducción de la puntuación promedio de las regiones tratadas en la escala de Sartorius de 18,8 a 17,2, con una reducción media en el DLQI del 6,4\%. Todos estos resultados se recogen en la tabla 1.

Terapia fotodinámica intralesional

Valladares Narganes et al. ${ }^{51}$ administraron ALA intralesional al $1 \%\left(0,2 \mathrm{ml} / \mathrm{cm}^{3}\right)$ tras irradiación, como se ha expuesto anteriormente, a 27 pacientes con un número total de una a 3 sesiones. Tras un seguimiento de 26,21 meses $(21,07-$ $68,57)$ se registraron 10 respuestas completas $(37 \%), 11$ respuestas moderadas (41\%) y 5 respuestas parciales $(19 \%)$, siendo la región axilar la que tuvo los mejores resultados (tabla 2).

Agut-Busquet et al. ${ }^{1}$ administraron una solución de AM al $1 \%$ intralesional, dirigida mediante ecografía en 7 de los pacientes, incubando durante 15 min e iluminando las lesiones con una lámpara de LED de $635 \mathrm{~nm}$ con una fluencia de $37 \mathrm{~J} / \mathrm{cm}^{2}$ en cada área (Aktilite ${ }^{\circledR}$ ). Tras un periodo de seguimiento de 6 meses, en un paciente la respuesta global fue del $78 \%$, en otro del $80 \%$, en 2 del $90 \%$ y en otros 2 de un $99 \%$. Los pacientes con Hurley tipo ॥ respondieron mejor que aquellos con Hurley tipo III (99 versus $87 \%$, respectivamente), y los mejores resultados se dieron en el área inguinal. Además, se apreció una mejoría significativa en el DLQI en 6 pacientes, coincidiendo con una excelente respuesta al tratamiento. En cuanto a los efectos adversos, fueron leves, ligera hinchazón o eritema, se resolvieron en una semana y estuvieron presentes en 6 pacientes (86\%) (tabla 2).

Suárez Valladares et al. ${ }^{50}$ administraron el ALA al $5 \%$ en gel por vía intralesional a través de una cánula en el trayecto de las fístulas en 38 pacientes. Tras incubación bajo oclusión opaca de $2 \mathrm{~h}$, se irradió con un láser de diodo intralesional de $630 \mathrm{~nm}$, con una sonda de fibra óptica de $400 \mathrm{~mm}(1,2 \mathrm{~W}$, fluencia de $180 \mathrm{~J} / \mathrm{cm}^{2}$ ). En todas las regiones tratadas se apreció una reducción en la puntuación de Sartorius modificada, objetivándose 53 respuestas completas, 7 buenas respuestas y 4 respuestas parciales, diferencias no estadísticamente significativas (tabla 2).

\section{Láser Nd:YAG}

Tierney et al. ${ }^{39}$ compararon el tratamiento con láser Nd:YAG más peróxido de benzoilo al $10 \%$ y clindamicina al $1 \%$ con aplicación tópica diariamente frente a solo el tratamiento tópico en 17 pacientes (tabla 1). Se administraron 4 sesiones a intervalos mensuales. Se obtuvo una reducción de la gravedad en la escala de Sartorius modificada del 65,3\% a los 3 meses en todos los sitios anatómicos (73,4\% inguinal, $62 \%$ axilar y $53,1 \%$ inframamaria), siendo estas mejorías estadísticamente significativas en todas las regiones tratadas con láser $(p<0,02)$ y no en las regiones control (solo con tratamiento tópico). 
$\mathrm{Xu}$ et al. ${ }^{45}$ realizaron un estudio prospectivo controlado en 19 pacientes con HS grado ॥ de Hurley que les trataron con 2 sesiones (mensuales) con Nd:YAG con una longitud de onda de $1.064 \mathrm{~nm}$. El porcentaje de respuesta en la escala de Sartorius tras 2 meses de tratamiento fue del 31,6\% ( $p=0,001)$, mejorando más en la zona inguinal (tabla 1).

En estadios muy precoces de la HS, en Hurley clase I, utilizando Nd:YAG, Zerbinati et al. ${ }^{47}$ obtuvieron en 12 pacientes una media de mejoría en el índice de Sartorius del 85,3\% (tabla 3). En esta misma línea, en 22 pacientes pero con enfermedad de gravedad superior a Hurley tipo ॥, Mahmoud et al. ${ }^{43}$ evalúan la respuesta del uso de 4 sesiones de Nd:YAG de pulso largo (una sesión mensual durante 4 meses), con un seguimiento de 6 meses, comprueban un descenso de la puntuación de Sartorius de $-72 \%$ (tabla 1 ).

\section{Otros tipos de láser}

Sobre el láser $\mathrm{CO}_{2}$, Abdel Azim et al. ${ }^{48}$ realizaron un ensayo en 20 pacientes mayores de 18 años con HS Hurley clase | 0 II, con el propósito de estudiar la seguridad y la eficacia del tratamiento combinado con láser fraccional $\mathrm{CO}_{2}$ y láser Nd:YAG de pulso largo $(1.064 \mathrm{~nm})$ en el tratamiento de la HS. Para ello, cada paciente recibió 4 sesiones de láser con un intervalo de 2 semanas: en el lado del tratamiento combinado, láser de $\mathrm{CO}_{2}$ fraccionado seguido de $\mathrm{Nd}$ :YAG de pulso largo $(1.064 \mathrm{~nm})$ en la misma sesión; y en el lado control, solo Nd:YAG de pulso largo $(1.064 \mathrm{~nm})$. Concluyeron que la combinación de ambos láseres consiguió un mayor grado de mejoría y satisfacción del paciente junto con una menor recurrencia en las primeras etapas (Hurley grado I y ॥) de la HS (tabla 3).

Madan et al. ${ }^{44}$, en una serie de 9 pacientes con HS refractaria a tratamiento médico y quirúrgico, los trataron con una sesión de láser $\mathrm{CO}_{2}$; a los 12 meses del tratamiento comprueban que 7 de los 9 pacientes tuvieron remisión completa, con alta satisfacción (tabla 1 ).

Por último, Fabbrocini et al. ${ }^{49}$ utilizan el láser de diodo en un grupo de 20 pacientes con HS Hurley estadio ।-॥ con PGA leve-moderada. Administrando 4 sesiones, una cada 2 semanas, registraron una reducción significativa del $31 \%$ en la puntuación de Sartorius, pasando de 28,55 $\pm 13,04$ a $19,75 \pm 12,29(p<0,05)$. Además, se observó una relación directa de la mejoría clínica con la mayor calidad de vida (tabla 3).

\section{Luz intensa pulsada}

Highton et al. ${ }^{11}$ llevaron a cabo un ensayo clínico aleatorizado en 18 pacientes comparando la aplicación del tratamiento en la región anatómica afecta por HS de un hemicuerpo con la contralateral, que no recibió tratamiento. El tratamiento consistió en la aplicación de IPL $\left(420 \mathrm{~nm}\right.$, fluencia $7-10 \mathrm{~J} / \mathrm{cm}^{2}$ a $\left.50 \mathrm{~ms}\right)$ y se observó una reducción promediada del $12 \%$ en la escala de Sartorius inmediatamente después de la terapia, del $10 \%$ a los 3 y 6 meses y del 3\% a los 12 meses. Se observó una importante diferencia entre el lado tratado y el lado control $(p<0,01)$. Hubo un alto nivel de satisfacción de los pacientes: una respuesta completa, 2 excelentes resultados, 10 buenos resultados y 4 resultados moderados. Quince no presentaron cambios significativos en el lado control, un participante informó de una ligera mejoría y otro un leve deterioro. El tratamiento fue aplicado una vez por semana, durante un periodo de 4 semanas, con un seguimiento de 12 meses (tabla 1).

\section{Discusión}

Considerando las limitaciones en la evidencia disponible sobre el tratamiento con luz en la HS, resulta difícil establecer la eficacia tanto con TFD intralesional como tópica, luz intensa pulsada y el láser Nd:YAG, ya que la comparación entre ellos no es fácil, dadas las importantes variaciones metodológicas.

En cuanto a la TFD, según esta revisión contamos con 3 fotosensibilizantes, ALA, MAL y AM, aplicados todos en distintas concentraciones, del 1 al 20\% para ALA, del 16\% para MAL y del 0,01 al 1\% para el AM. Es difícil establecer qué concentración ofrece una tasa de respuesta mayor, dada la gran heterogeneidad de las medidas de resultado. Además, el tipo de luz utilizada para la irradiación en cada estudio también difiere, ya que se utilizan lámparas $\operatorname{LED}^{1,40,42}$, láseres, ya sean externos ${ }^{2}$ o intralesionales ${ }^{50,51}$, e $\mathrm{IPL}^{38,41}$, con francas diferencias en su penetración y, por tanto, en su posibilidad de tratar las lesiones profundas de la HS, un factor importante en la eficacia. Además, también son diferentes la dosis y la intensidad de la luz aplicada. Sin embargo, la longitud de onda es la misma: 630-635 nm.

Asimismo, se observan diferencias en cuanto al área tratada, la duración y el número de sesiones, desde una ${ }^{50}$ hasta $12^{38}$. La principal distinción con respecto a esta técnica es el uso de forma tópica o intralesional tanto del fotosensibilizante (ALA, MAL o AM) como de la luz (LED, láser o luz intensa pulsada).

Con respecto a la TFD intralesional, la técnica empleada difiere entre autores. Suárez Valladares et al. ${ }^{50}$ y ValladaresNarganes et al. ${ }^{51}$ utilizan láser de diodo de $630 \mathrm{~nm}$ y ALA a diferentes concentraciones, ambos intralesionales, y evalúan la respuesta con la escala de Sartorius modificada, encontrando una mayor respuesta utilizando ALA al 5\% en gel y láser de diodo de $400 \mathrm{~nm}, 1,2 \mathrm{~W}$ y $180 \mathrm{~J}$. Por otro lado, Agut-Busquet et al. ${ }^{1}$ utilizan AM al $1 \%$ y una lámpara de LED de $635 \mathrm{~nm}$ y fluencia de $37 \mathrm{~J} / \mathrm{cm}^{2}$, y evalúan los resultados mediante el DQLI y la escala Sartorius, obteniendo una respuesta que va desde un $99 \%$ en la etapa II de Hurley a un $87 \%$ en la etapa III.

Con respecto a la IPL, solo hay un estudio disponible ${ }^{11}$, que encuentra una mejoría estadísticamente significativa que puede mantenerse hasta 12 meses, además, con riesgo bajo de complicaciones.

En el caso del uso del láser de Nd:YAG, el ensayo clínico controlado y aleatorizado de Tierney et al. ${ }^{39}$ demuestra su efectividad; posteriormente se han ido publicando series de casos en los que se demuestra una mejora en el número de brotes y la gravedad ${ }^{43,47}$, superior en la región inguinal ${ }^{45}$.

Muy pocos son los estudios con otros tipos de láser, pero todos obtienen resultados muy esperanzadores; se ha comparado el láser $\mathrm{CO}_{2}$ en combinación con $\mathrm{Nd}$ :YAG frente a solo tratamiento con el láser $\mathrm{Nd}$ :YAG, obteniendo mejores resultados con la combinación y una mayor satisfacción del paciente ${ }^{48}$. Madan et al. ${ }^{44}$ comprobaron que con el uso en monoterapia del láser $\mathrm{CO}_{2}$ y marsupialización también se obtienen altas tasas de remisión completa, pese a los efectos 
secundarios que recogen, como es la cicatrización hipertrófica.

Solo se ha incluido un estudio con láser diodo en pacientes con HS y PGA leve-moderada en el que parece que existe una mejoría clínica y también en la calidad de vida ${ }^{48}$.

En conclusión, la evidencia para el uso de los tratamientos con luz en la HS es limitada, pudiendo separar los tratamientos en 2 grandes grupos en función de si se usa fotosensibilizante o no: un grupo con calidad de evidencia baja-moderada que incluye el uso de láser Nd:YAG, $\mathrm{CO}_{2}$ o IPL; y otro grupo con fotosensibilizante con calidad de evidencia de baja-moderada, con más estudios de calidad moderada utilizando la TFD intralesional frente a la TFD tópica. Aunque todos los procedimientos se engloban en tratamientos con luz, cada uno de los grupos no es comparable entre sí, ya que tienen diferente mecanismo de acción, pero al evaluar la eficacia de cada uno parece que aquellos procedimientos que permiten una mayor penetración del tratamiento, ya sea la TFD intralesional o los láseres, obtienen los mejores resultados.

Se necesitan ensayos clínicos controlados y aleatorizados que confirmen la eficacia de cada uno de estos tratamientos y estandaricen el protocolo más adecuado para poder garantizar un adecuado nivel de evidencia en términos de eficacia y seguridad.

\section{Autoría}

T. Gracia Cazaña y L.V. Berdel García han contribuido de la misma manera a la realización del artículo.

\section{Agradecimientos}

Agradecemos a D. ${ }^{a}$ Visitación Ortega Riba y a D. ${ }^{a}$ Montserrat Salas Valera su ayuda en la realización de la búsqueda sistemática y la obtención de artículos.

Nuestro agradecimiento también al Grupo de Investigación reconocido por el Gobierno de Aragón B18_17D.

\section{Anexo. Material adicional}

Se puede consultar material adicional a este artículo en su versión electrónica disponible en doi:10.1016/ j.ad.2019.04.008.

\section{Bibliografía}

1. Agut-Busquet E, Romaní J, Gilaberte Y, García-Malinis A, RiberaPibernat M, Luelmo J. Photodynamic therapy with intralesional methylene blue and a $635 \mathrm{~nm}$ light-emitting diode lamp in hidradenitis suppurativa: A retrospective follow-up study in 7 patients and a review of the literature. Photochem Photobiol Sci. 2016;15:1020-8.

2. Andino Navarrete R, Hasson Nisis A, Parra Cares J. Efectividad de la terapia fotodinámica con ácido 5-aminolevulínico en el tratamiento de la hidrosadenitis supurativa. Serie de 5 casos. Actas Dermosifiliogr. 2014;105:614-7.

3. Ingram JR, Woo PN, Chua SL, Ormerod AD, Desai N, Kai AC, et al. Interventions for hidradenitis suppurativa. Cochrane Database Syst Rev. 2015;10:CD010081.
4. Herane MI, Alarcón R. Hidrosadenitis supurativa y procesos afines. Mas Dermatol. 2012;16:4-12.

5. García-Martínez FJ, Pascual JC, López-Martín I, PereyraRodríguez JJ, Martorell Calatayud A, Salgado-Boquete L, et al. Actualización en hidrosadenitis supurativa en Atención Primaria. Semergen. 2017;43:34-42.

6. Martorell A, García-Martínez FJ, Jiménez-Gallo D, Pascual JC. Actualización en hidradenitis supurativa (I): epidemiología, aspectos clínicos y definición de severidad de la enfermedad. Actas Dermosifiliogr. 2015;106:703-15.

7. Buggiani G, Troiano M, Rossi R, Lotti T. Photodynamic therapy: Off-label and alternative use in dermatological practice. Photodiagnosis Photodyn Ther. 2008;5:134-8.

8. MacCormack MA. Photodynamic therapy in dermatology: An update on applications and outcomes. Semin Cutan Med Surg. 2008;27:52-62.

9. Dunn J, Koo J. Psychological stress and skin aging: A review of possible mechanisms and potential therapies. Dermatol Online J. 2013;19, 18561185619.

10. Piccolo D, di Marcantonio D, Crisman G, Cannarozzo G, Sannino M, Chiricozzi A, et al. Unconventional use of intense pulsed light. Biomed Res Int. 2014;2014, 6182066182110.

11. Highton L, Chan WY, Khwaja N, Laitung JKG. Treatment of hidradenitis suppurativa with intense pulsed light: A prospective study. Plast Reconstr Surg. 2011; $128: 459-65$

12. Saunte DM, Lapins J. Lasers and intense pulsed light hidradenitis suppurativa. Dermatol Clin. 2016;34:111-9.

13. Jadad AR, Moore RA, Carroll D, Jenkinson C, Reynolds DJ, Gavaghan DJ, et al. Assessing the quality of reports of randomized clinical trials: Is blinding necessary? Control Clin Trials. 1996; $17: 1-12$

14. Schulz KF, Altman DG, Moher D, CONSORT Group. CONSORT 2010 statement: Updated guidelines for reporting parallel group randomised trials. Int J Surg. 2011;9:672-7.

15. López de Argumedo M, Reviriego E, Andrío E, Rico R, Sobradillo $N$, Hurtado de Saracho I. Revisión externa y validación de instrumentos metodológicos para la Lectura Crítica y la síntesis de la evidencia científica. Madrid: Plan Nacional para el SNS del MSC, Servicio de Evaluación de Tecnologías Sanitarias del País Vasco (Osteba); 2006.

16. Silva-Feistner M, Salomone C, Silva S, Vial-Letelier V. [Prepubertal hidradenitis suppurativa: Report of 2 clinical cases] Spanish. Rev Chil Pediatr. 2016;87:193-8.

17. Ingram JR. Hidradenitis suppurativa: An update. Clin Med (Lond). 2016;16:70-3.

18. Grimstad $\varnothing$, Ingvarsson $G$. Carbon dioxide laser treatment using methylene blue-assisted sinus tract identification in hidradenitis suppurativa. Dermatologic Surg. 2016;42:1303-4.

19. Suárez-Valladares MJ, Rodriguez-Prieto MÁ, Serra-Llusà R. Penetration of $630 \mathrm{~nm}$ laser and 5-aminolevulinic acid in tissue with intralesional photodynamic therapy. Photodiagnosis Photodyn Ther. 2016;16:166-8.

20. Nicholson CL, Hamzavi I, Ozog DM. Rapid healing of chronic ulcerations and improvement in range of motion after fractional carbon dioxide $\left(\mathrm{CO}_{2}\right)$ treatment after $\mathrm{CO}_{2}$ excision of hidradenitis suppurativa axillary lesions: A case report. JAAD Case Rep. 2016;2:4-6.

21. Chen W, Plewig G. Should hidradenitis suppurativa/acne inversa best be renamed as "dissecting terminal hair folliculitis"? Exp Dermatol. 2017;26:544-7.

22. Gulliver W, Zouboulis CC, Prens E, Jemec GB, Tzellos T. Evidence-based approach to the treatment of hidradenitis suppurativa/acne inversa, based on the European guidelines for hidradenitis suppurativa. Rev Endocr Metab Disord. 2016;17:343-51.

23. Ingram JR, Woo PN, Chua SL, Ormerod AD, Desai N, Kai AC, et al. Interventions for hidradenitis suppurativa: A Cochrane 
systematic review incorporating GRADE assessment of evidence quality. Br J Dermatol. 2016;174:970-8.

24. Van Rappard DC, Mekkes JR, Tzellos T. Randomized controlled trials for the treatment of hidradenitis suppurativa. Dermatol Clin. 2016;34:69-80.

25. Mordon S. Treating hidradenitis suppurativa with photodynamic therapy. J Cosmet Laser Ther. 2018;20:223-8.

26. Zhang Y, Yang Y, Zou X. Photodynamic therapy for hidradenitis suppurativa/acne inversa: Case report. Photodiagnosis Photodyn Ther. 2018;22:251-2.

27. Jourabchi N, Anzaldi L, Erdag G, Cohen B. Pulsed-dye laser treatment of a nonhealing chronic wound that developed after incision and drainage of a hidradenitis suppurativa abscess. Dermatol Surg. 2018;44:447-9.

28. Scuderi N, Monfrecola A, Dessy LA, Fabbrocini G, Megna M, Monfrecola G. Medical and surgical treatment of hidradenitis suppurativa: A review. Skin Appendage Disord. 2017;3:95-110.

29. Emtestam L, Lapins J, Sartorius K. Carbon dioxide laser treatment using methylene blue-assisted sinus tract identification in hidradenitis suppurativa. Dermatologic Surg. 2017;43:604-5.

30. Ingram JR. Interventions for hidradenitis suppurativa. JAMA Dermatol. 2017;153:458.

31. Levoska MA, Nicholson CL, Hamzavi IH. A retrospective review of light- and laser-based management of hidradenitis suppurativa. Semin Cutan Med Surg. 2017;36:67-74.

32. Hamzavi IH, Griffith JL, Riyaz F, Hessam S, Bechara FG. Laser and light-based treatment options for hidradenitis suppurativa. J Am Acad Dermatol. 2015;73:S78-81.

33. Marasca C, Annunziata MC, Napolitano M, Fabbrocini G. Unconventional therapies for hidradenitis suppurativa. Expert Rev Clin Pharmacol. 2018;11:879-87.

34. Zhang L, Wang P, Shi L, Zhang G, Zhang Y, Zhou Z, et al. Topical 5aminolevulinic acid photodynamic therapy improved refractory acne conglobata and perifolliculitis capitis abscedens et suffodiens rather than hidradenitis suppurativa. J Innov Opt Health Sci. 2016;9:1640002.

35. Mikkelsen PR, Dufour DN, Zarchi K, Jemec GB. Recurrence rate and patient satisfaction of $\mathrm{CO}_{2}$ laser evaporation of lesions in patients with hidradenitis suppurativa: A retrospective study. Dermatol Surg. 2015;41:255-60.

36. Vossen ARJV, van der Zee HH, Terian M, van Doorn MBA, Prens EP. Laser hair removal alters the disease course in mild hidradenitis suppurativa. J Dtsch Dermatol Ges. 2018;16:901-3.

37. Theut Riis P, Saunte DM, Sigsgaard V, Wilken C, Jemec GB. Intense pulsed light treatment for patients with hidradenitis suppurativa: Beware treatment with resorcinol. J Dermatolog Treat. 2018;29:385-7.

38. Fadel MA, Tawfik AA. New topical photodynamic therapy for treatment of hidradenitis suppurativa using methylene blue niosomal gel: A single-blind, randomized, comparative study. Clin Exp Dermatol. 2015;40:116-22.

39. Tierney E, Mahmoud BH, Hexsel C, Ozog D, Hamzavi I. Randomized control trial for the treatment of hidradenitis suppurativa with a neodymium-doped yttrium aluminium garnet laser. Dermatol Surg. 2009;35:1188-98.

40. Calzavara-Pinton PG, Rossi MT, Aronson E, Sala R, Italian Group For Photodynamic Therapy. A retrospective analysis of real-life practice of off-label photodynamic therapy using methyl ami- nolevulinate (MAL-PDT) in 20 Italian dermatology departments. Part 1: Inflammatory and aesthetic indications. Photochem Photobiol Sci. 2013;12:148-57.

41. Schweiger ES, Riddle CC, Aires DJ. Treatment of hidradenitis suppurativa by photodynamic therapy with aminolevulinic acid: Preliminary results. J Drugs Dermatol. 2011;10:381-6.

42. Sotiriou E, Apalla Z, Maliamani F, loannides D. Treatment of recalcitrant hidradenitis suppurativa with photodynamic therapy: Report of five cases. Clin Exp Dermatol. 2009;34: e235-6.

43. Mahmoud BH, Tierney E, Hexsel CL, Pui J, Ozog DM, Hamzavi $\mathrm{IH}$. Prospective controlled clinical and histopathologic study of hidradenitis suppurativa treated with the long-pulsed neodymium:yttrium-aluminium-garnet laser. J Am Acad Dermatol. 2010;62:637-45.

44. Madan V, Hindle E, Hussain W, August PJ. Outcomes of treatment of nine cases of recalcitrant severe hidradenitis suppurativa with carbon dioxide laser. Br J Dermatol. 2008;159:1309-14.

45. Xu LY, Wright DR, Mahmoud BH, Ozog DM, Mehregan DA, Hamzavi $\mathrm{IH}$. Histopathologic study of hidradenitis suppurativa following long-pulsed 1064-nm Nd:YAG laser treatment. Arch Dermatol. 2011;147:21-8.

46. Suárez Valladares MJ, Eiris Salvado N, Rodríguez Prieto MA. Treatment of hidradenitis suppurativa with intralesional photodynamic therapy with 5-aminolevulinic acid and $630 \mathrm{~nm}$ laser beam. J Dermatol Sci. 2017;85:241-6.

47. Zerbinati N, D’Este E, Ini L, Baruffato A, Premoli V, Calligaro A, et al. Clinical and histological changes in hidradenitis suppurativa following $1064 \mathrm{~nm}$ nd:YAG intralesional laser treatment. J Biol Regul Homeost Agents. 2017;31:131-40.

48. Abdel Azim AA, Salem RT, Abdelghani R. Combined fractional carbon dioxide laser and long-pulsed neodymium?: yttriumaluminium-garnet $(1064 \mathrm{~nm})$ laser in treatment of hidradenitis suppurativa; a prospective randomized intra-individual controlled study. Int J Dermatol. 2018;57:1135-44.

49. Fabbrocini G, França K, Lotti T, Marasca C, Annunziata MC, Cacciapuoti S, et al. Intralesional diode laser $1064 \mathrm{~nm}$ for the treatment of hidradenitis suppurativa: A report of twenty patients. Open Access Maced J Med Sci. 2018;6:31-4.

50. Suárez Valladares MJ, Salvado NE, Rodríguez Prieto MÁ. Treatment of hidradenitis suppurativa with intralesional photodynamic therapy with 5-aminolevulinic acid and $630 \mathrm{~nm}$ laser beam. 2017;85:241-6.

51. Valladares-Narganes LM, Rodríguez-Prieto MA, Blanco-Suárez MD, Rodriguez-Lage C, García-Doval I. Treatment of hidradenitis suppurativa with intralesional photodynamic therapy using a laser diode attached to an optical cable: A promising new approach. Br J Dermatol. 2015;172:1136-9.

52. John H, Manoloudakis N, Stephen Sinclair J. A systematic review of the use of lasers for the treatment of hidradenitis suppurativa. J Plast Reconstr Aesthet Surg. 2016;69: 1374-81.

53. Robert E, Bodin F, Paul C, Konstantinou MP, Gall Y, Grolleau $\mathrm{J}$-L, et al. Non-surgical treatments for hidradenitis suppurativa: A systematic review. Ann Chir Plast Esthet. 2017;62: 274-94. 